\title{
INTEGRAL EQUATIONS ASSOCIATED WITH HANKEL CONVOLUTIONS $\left({ }^{1}\right)$
}

\author{
BY \\ DEBORAH TEPPER HAIMO \\ Chapter I. Introduction
}

1. Objectives. We denote by $V\left[a_{1}, \cdots, a_{n}\right]$ the number of variations of sign of the sequence $a_{1}, \cdots, a_{n}$ of real numbers. Hence, for example, $V[-1,0,1]=1, V[1,0,1]=0, V[0,0,0]=-1$. If $f$ is a real function defined for $0<x<\infty$, then $V[f(x)]=$ l.u.b. $V\left[f\left(x_{1}\right), \cdots, f\left(x_{n}\right)\right]$, taken over all sets $0<x_{1}<x_{2}<\cdots<\infty$.

Let $H$ be a real-valued function of $L^{1}(-\infty, \infty), \phi$ a real-valued, continuous function of $L^{\infty}(-\infty, \infty)$ and let

$$
H * \phi(x)=\int_{-\infty}^{\infty} H(x-t) \phi(t) d t, \quad-\infty<x<\infty .
$$

$H$ is said to be a variation diminishing *-kernel if and only if, for every such $\phi$,

$$
V[H * \phi(x)] \leqq V[\phi(x)]
$$

Let

$$
G(t)=\frac{1}{2 \pi} \int_{-\infty}^{\infty} \frac{e^{i s t}}{E(s)} d s, \quad-\infty<t<\infty,
$$

where

$$
E(s)=e^{c s^{2}+i b s} \coprod_{k=1}^{\infty}\left(1-i \frac{s}{a_{k}}\right) e^{i s / a_{k}}
$$

the $a_{k}$ 's being real, with $\sum_{k=1}^{\infty}\left(1 / a_{k}^{2}\right)<\infty$, and $b$ and $c$ real, with $c \geqq 0$. In 1947 , I. J. Schoenberg proved that the kernel $G$ defined by (3) is vari-

Presented to the Society, November 4, 1963 under the title Inversion for Hankel convolutions, and January 25, 1964 under the title Representation for Hankel convolutions; received by the editors November 5,1963 .

( $\left.{ }^{1}\right)$ This paper includes the major part of the author's doctoral dissertation at Harvard University. Thanks are due to Professor I. I. Hirschman, Jr., of Washington University who suggested the problem and who gave invaluable guidance. The research was supported, in part, under a National Science Foundation contract at Washington University. The typing of the manuscript was subsidized partially by Southern Illinois University. 
ation diminishing and that, conversely, every variation diminishing kernel is of the form (3); see [13].

The following analogous theory, generalizing the kernels of (3), was developed by I. I. Hirschman, Jr. [5]:

Let $\gamma$ be a fixed positive number, and set

$$
\mu(x)=\frac{x^{2 \gamma+1}}{2^{\gamma+1 / 2} \Gamma(\gamma+3 / 2)} .
$$

Define

$$
\mathscr{f}(x)=2^{\gamma-1 / 2} \Gamma(\gamma+1 / 2) x^{1 / 2-\gamma} J_{\gamma-1 / 2}(x),
$$

where $J_{\gamma-1 / 2}(x)$ is the ordinary Bessel function of order $\gamma-1 / 2$. Replacing the exponential factor of the integrand on the right of (3) by $\mathscr{f}(s t)$, we set

$$
G(t)=\int_{0}^{\infty} \frac{\mathscr{J}(s t)}{E(s)} d \mu(s), \quad-\infty<t<\infty,
$$

where

$$
E(s)=e^{c s^{2}} \prod_{k=1}^{\infty}\left(1+\frac{s^{2}}{a_{k}^{2}}\right),
$$

the $a_{k}$ 's being real, $\sum_{k=1}^{\infty}\left(1 / a_{k}^{2}\right)<\infty$, and $c \geqq 0$. Further, corresponding to

$$
G(x-y)=\frac{1}{2 \pi} \int_{-\infty}^{\infty} \frac{\exp [i s x] \exp [\overline{i s y}]}{E(s)} d s,
$$

we set

$$
G(x, y)=\int_{0}^{\infty} \frac{\mathscr{f}(s x) \mathscr{J}(s y)}{E(s)} d \mu(s),
$$

and define, for $\phi$ in $L^{1}(0, \infty)$,

$$
G \# \phi(x)=\int_{0}^{\infty} G(x, t) \phi(t) d \mu(t), \quad 0<x<\infty .
$$

A general definition of $\psi \# \phi(x)$ for any two functions $\psi$ and $\phi$ of $L^{1}\left(0, \infty ; d_{\mu}(x)\right)$ will be given in II, §2. Hirschman proved that a \#-kernel $G$ is variation diminishing if and only if it has the form (7). We note that in the special case where $\gamma=0, \mathscr{f}(x)=\cos x$, and we have Schoenberg's theorem for an even kernel.

In 1955 , I. I. Hirschman, Jr. and D. V. Widder showed that the *convolution transform $f(x)=G * \phi(x)$ can be inverted by the differential operator $E(D)$ : 


$$
E(D) f(x)=\lim _{N \rightarrow \infty} e^{b D} \prod_{k=1}^{N}\left(1-\frac{D}{a_{k}}\right) e^{D_{i k}} f(x)=\phi(x),
$$

where $D$ stands for differentiation with respect to $x$. Further, they obtained a corresponding representation theory, [10, pp. 120-169].

These results suggest that we may develop a parallel inversion theory for the \#-convolution of (11). Our primary goal will be to derive such a theory under the least restrictive assumptions and to establish an analogous representation theory. Our main inversion theorem will be the following:

Let $\phi$ be a function integrable on every finite interval and let

$$
f(x)=\int_{0}^{\infty} G(x, t) \phi(t) d \mu(t), \quad 0<x<\infty,
$$

where

$$
G(x, y)=\int_{0}^{\infty} \frac{\mathscr{J}(x t) \not{f}(y t)}{\prod_{k=1}^{\infty}\left(1+\frac{t^{2}}{a_{k}^{2}}\right)} d \mu(t)
$$

the $a_{k}$ 's being real, $0<a_{1}<\ldots<\infty$, with $\sum_{k=1}^{\infty}\left(1 / a_{k}^{2}\right)<\infty$. Then

$$
\lim _{N \rightarrow \infty} \prod_{k=1}^{N}\left(1-\frac{\Delta_{x}}{a_{k}^{2}}\right) f(x)=\phi(x),
$$

where $\Delta_{x} h(x)=h^{\prime \prime}(x)+2 \gamma h^{\prime}(x) / x$, if $\lim _{h \rightarrow 0} 1 / h \int_{x}^{x+h}[\phi(t)-\phi(x)] d \mu(t)=0$, a condition which holds a.e.

Correspondingly, we prove that necessary and sufficient conditions for a function $f$ to be represented by $f(x)=\int_{0}^{\infty} G(x, t) d \psi(t)$ with $\psi(t) \uparrow$ are that $f(x) \in C^{\infty}, 0 \leqq x<\infty, \prod_{k=1}^{N_{i}}\left(1-\Delta_{x} / a_{k}^{2}\right) f(x) \geqq 0,0 \leqq x<\infty, 1=N_{0}$ $<N_{1}<\cdots, f(x)=o\left(\mathscr{P}\left(a_{1} x\right)\right), x \rightarrow \infty$, where $\mathscr{P}\left(a_{1} x\right)$ is defined in III, $\$ 3,(3)$.

Examples which serve to illustrate these results are given in the following table:

\begin{tabular}{l|lc}
$\gamma$ & $E(t)$ & $G(x, y)$ \\
\hline 0 & $\cosh (t / 2)$ & $(2 \pi)^{1 / 2} \frac{\cosh \pi x \cosh \pi y}{\cosh ^{2} \pi x+\sinh ^{2} \pi y}$ \\
0 & $\frac{\sinh \pi t}{\pi t}$ & $(\pi / 2)^{1 / 2} \frac{\cosh x \cosh y+1}{(\cosh x+\cosh y)^{2}}$ \\
1 & $\frac{(2 \pi)^{1 / 2}}{x y} \frac{\sinh \pi x \sinh \pi y}{\sinh ^{2} \pi x+\cosh ^{2} \pi y}$ \\
1 & $\frac{\cosh (t / 2)}{\pi t}$ & $(\pi / 2)^{1 / 2} \frac{1}{x y} \frac{\sinh x \sinh y}{(\cosh x+\cosh y)^{2}}$
\end{tabular}.


Note that the Stieltjes transform

$$
F(x)=\int_{0}^{\infty} \frac{\Phi(t)}{x+t} d t
$$

becomes, after exponential changes of variables,

$$
h(x)=\int_{-\infty}^{a} \frac{\psi(t)}{2 \cosh \frac{x-t}{2}} d t,
$$

where $h(t)=F\left(e^{t}\right) e^{t / 2}, \psi(t)=e^{t / 2} \Phi\left(e^{t}\right)$.

Hence, if $\psi$ is an even function, we have

$$
\begin{aligned}
h(x) & =\frac{1}{2} \int_{0}^{\infty}\left[\frac{1}{\cosh \frac{x+t}{2}}+\frac{1}{\cosh \frac{x-t}{2}}\right] \psi(t) d t \\
& =2 \pi \int_{0}^{\infty} \frac{\cosh \pi x \cosh \pi t}{\cosh ^{2} \pi x+\cosh ^{2} \pi t} \psi(2 \pi t) d t,
\end{aligned}
$$

or

$$
f(x)=(2 / \pi)^{1 / 2} \int_{0}^{\infty}(2 \pi)^{1 / 2} \frac{\cosh \pi x \cosh \pi t}{\cosh ^{2} \pi x+\cosh ^{2} \pi t} \phi(t) d t,
$$

where $f(x)=h(x) / \pi, \phi(x)=\psi(2 \pi x)$. Thus we find that the Stieltjes transform for $\Phi$ satisfying $\Phi(1 / x)=x \Phi(x)$ is the special case of the \#-convolution illustrated by our first example above. The inversion theorem enables us to conclude that, in this case,

$$
\lim _{N \rightarrow \infty} \prod_{k=1}^{N}\left[1-\frac{1}{(2 k-1)^{2} \pi^{2}} \frac{d^{2}}{d x^{2}}\right] f(x)=\phi(x),
$$

see $[10$, p. 69].

2. Formal approach. Before proceeding to a rigorous development, let us, by way of illustration, derive the inversion theorem formally. We consider a real-valued function $f$ defined on $(0, \infty)$ and set

$$
f^{\hat{f}}(x)=\int_{0}^{\infty} \mathcal{f}(x t) f(t) d \mu(t), \quad 0<x<\infty,
$$

so that, by inversion, as in the case of the Fourier transform, we have

$$
f(x)=\int_{0}^{\infty} \not{f}(x t) f^{\hat{\gamma}}(t) d \mu(t) .
$$

We define the linear differential operator $\Delta_{x}$ by 


$$
\left(\Delta_{x} f\right) x=\left[\frac{d^{2}}{d x^{2}}+\frac{2 \gamma}{x} \frac{d}{d x}\right] f(x) .
$$

By an application of Bessel's equation, we may show that, for $t$ fixed,

$$
\Delta_{x} f(x t)=-t^{2} \mathscr{f}(x t)
$$

see $[5$, p. 317]. Note the analogy to the Fourier transform where the derivative operator $D$ applied to the exponential gives $D\left(e^{i t x}\right)=i t e^{i t x}$. Next, let

$$
E(s)=\prod_{k=1}^{\infty}\left[1+\frac{s^{2}}{a_{k}^{2}}\right]
$$

where the $a_{k}$ 's are real and $\sum_{k=1}^{\infty}\left(1 / a_{k}^{2}\right)<\infty$. Further, for a real-valued function $\phi$ defined on $(0, \infty)$, let

$$
f(x)=\int_{0}^{\infty} G(x, y) \phi(y) d \mu(y), \quad 0<x<\infty,
$$

where $G(x, y)$ is defined in $\$ 1,(10)$. It then follows formally that

$$
\begin{aligned}
f^{\prime}(x) & =\int_{0}^{\infty} f(t) \mathscr{f}(x t) d \mu(t) \\
& =\int_{0}^{\infty} \mathscr{f}(x t) d \mu(t) \int_{0}^{\infty} G(t, y) \phi(y) d \mu(y) \\
& =\int_{0}^{\infty} \phi(y) d \mu(y) \int_{0}^{\infty} \mathscr{f}(x t) G(t, y) d \mu(t) \\
& =\int_{0}^{\infty} \phi(y) \frac{\mathcal{J}(x y)}{E(x)} d \mu(y) \\
& =\frac{\phi(x)}{E(x)} .
\end{aligned}
$$

Hence

$$
f(x)=\int_{0}^{\infty} \not{f}(x t) f^{\hat{\prime}}(t) d \mu(t)=\int_{0}^{\infty} \not{f}(x t) \frac{\hat{\phi}(t)}{E(t)} d_{\mu}(t) .
$$

We thus find that

$$
\begin{aligned}
\prod_{k=1}^{N}\left[1-\frac{\Delta_{x}}{a_{k}^{2}}\right] f(x) & =\int_{0}^{\infty}\left\{\prod_{k=1}^{N}\left[1-\frac{\Delta_{x}}{a_{k}^{2}}\right] \mathscr{f}(x t)\right\} \frac{\phi^{\hat{(}}(t)}{E(t)} d_{\mu}(t) \\
& =\int_{0}^{\infty} \frac{\mathscr{f}^{\prime}(x)^{-}(t)}{\prod_{k=N+1}^{\infty}\left[1+\frac{t^{2}}{a_{k}^{2}}\right]} d \mu(t) .
\end{aligned}
$$


It, therefore, follows that

$$
\begin{aligned}
& \lim _{N \rightarrow \infty} \prod_{k=1}^{N}\left[1-\frac{\Delta_{x}}{a_{k}^{2}}\right] f(x)=\int_{0}^{\infty} \frac{\not{f}(x t) \phi^{\hat{n}}(t)}{\lim _{N \rightarrow \infty} \prod_{k=N+1}^{\infty}\left[1+\frac{t^{2}}{a_{k}^{2}}\right]} d \mu(t) \\
& =\int_{0}^{\infty} \not(x t) \phi^{\hat{\gamma}}(t) d \mu(t) \\
& =\phi(x) \text {, }
\end{aligned}
$$

which is the inversion we seek.

Chapter II. Definitions AND PREliminary Results

1. Basic definitions. We begin by developing the theory of the Hankel transform and by establishing some of the properties analogous to those of the Fourier transform. For fixed $\gamma>0$, we define

$$
d \mu(x)=\frac{x^{2 \gamma}}{2^{\gamma-1 / 2} \Gamma(\gamma+1 / 2)} d x .
$$

We denote by $L^{p}(0, \infty), 1 \leqq p<\infty$, the space of all real-valued, measurable functions $f$ defined on $(0, \infty)$ with norm

$$
\|f\|_{p}=\left[\int_{0}^{\infty}|f(x)|^{p} d \mu(x)\right]^{1 / p}<\infty,
$$

whereas $L^{\infty}(0, \infty)$ denotes the space of those functions $f$ for which

$$
\|f\|_{\infty}=\underset{0<x<\infty}{\operatorname{ess}} \text { l.u.b. }|f(x)|<\infty .
$$

Let $\Delta(x, y, z)$ be the area of a triangle with sides $x, y, z$ if such a triangle exists. Set

$$
D(x, y, z)=\frac{2^{3 \gamma-5 / 2}[\Gamma(\gamma+1 / 2)]^{2}}{\Gamma(\gamma) \pi^{1 / 2}}(x y z)^{-2 \gamma+1}[\Delta(x, y, z)]^{2 \gamma-2}
$$

if $\Delta$ exists and zero otherwise. We note that $D(x, y, z) \geqq 0$ and that $D(x, y, z)$ is symmetric in $x, y, z$. Further, we have the following basic formula:

$$
\int_{0}^{\infty} \mathscr{f}(z t) D(x, y, z) d \mu(z)=\mathscr{f}(x t) \not{f}(y t),
$$

[17, p. 411], from which it follows immediately, on setting $t=0$, that

$$
\int_{0}^{\infty} D(x, y, z) d \mu(z)=1
$$

Using (5), we may show that 


$$
|\not f(x)| \leqq 1, \quad 0 \leqq x<\infty ;
$$

see $[5$, p. 310].

2. Preliminary results. For each $f$ in $L^{1}(0, \infty)$, it is clear, by $\$ 1$, (7), that the integral $\int_{0}^{\infty} \mathscr{f}(x t) f(t) d \mu(t)$ exists, so that we may define the Hankel transform $f^{\wedge}$ of a function $f$ in $L^{1}(0, \infty)$ by

$$
f^{\hat{\prime}}(x)=\int_{0}^{\infty} \mathscr{f}(x t) f(t) d \mu(t) .
$$

Lemma 2.1. Let $f$ be a function of $L^{1}(0, \infty)$. Then $f^{\wedge}(x)$ is bounded and continuous for $0 \leqq x<\infty$.

Proof. Since

$$
\left|f^{\hat{\prime}}(x)\right| \leqq \int_{0}^{\infty}|\not \mathscr{f}(x t) f(t)| d \mu(t) \leqq\|f\|_{1}
$$

we have

$$
\underset{0 \leqq x<\infty}{\operatorname{l.u.b} .}\left|f^{\hat{n}}(x)\right| \leqq\|f\|_{1}
$$

and the transform is clearly bounded.

Further, the continuity of $f^{n}(x)$ for $0 \leqq x<\infty$ may be established by noting that, for any real $x$ and $h, 0 \leqq x<\infty$, we have

$$
\left|f^{\wedge}(x+h)\right| \leqq \int_{0}^{\infty}|f((x+h) t)||f(t)| d \mu(t) .
$$

Now $|\not f((x+h) t)||f(t)| \leqq|f(t)|$ and $\lim _{h \rightarrow 0}|\not f((x+h) t)|=|\mathscr{f}(x t)|$, $0 \leqq t<\infty$, so that the Lebesgue convergence theorem may be applied and the result follows.

LEMma 2.2. Let $f, f_{1}, f_{2}, \ldots$ be a sequence of functions of $L^{1}(0, \infty)$ such that

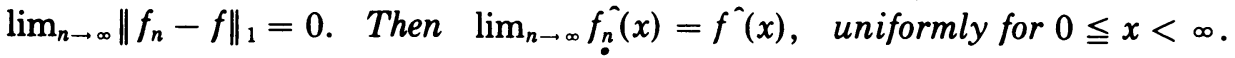

Proof. By Lemma 2.1, (2), we have

$$
\left|f_{n}^{\hat{n}}(x)-f^{\hat{\prime}}(x)\right| \leqq\left\|f_{n}-f\right\|_{1} \text {. }
$$

Definition 2.3. The associated function $f(x, y)$ corresponding to a function $f(x)$ of $L^{1}(0, \infty)$ is given by

$$
f(x, y)=\int_{0}^{\infty} f(u) D(x, y, u) d \mu(u), \quad 0<x, y<\infty .
$$

The following lemma justifies the definition.

Lemma 2.4. Let $f$ be a function of $L^{1}(0, \infty)$. Then the integral

$$
\int_{0}^{\infty} f(u) D(x, y, u) d \mu(u)
$$


converges for almost all $y$ for each fixed $x$, and, for $x$ fixed, $\|f(x, \cdot)\|_{1} \leqq\|f\|_{1}$.

Proof. We have

$$
\begin{aligned}
\int_{0}^{\infty} \mid \int_{0}^{\infty} & f(u) D(x, y, u) d_{\mu}(u) \mid d_{\mu}(y) \\
& \leqq \int_{0}^{\infty} d_{\mu}(y) \int_{0}^{\infty}|f(u)| D(x, y, u) d_{\mu}(u) \\
& =\int_{0}^{\infty}|f(u)| d_{\mu}(u) \int_{0}^{\infty} D(x, y, u) d_{\mu}(y) \\
& =\|f\|_{1},
\end{aligned}
$$

and hence $f(x, y)$ exists for almost all $y$, for each fixed $x$, and vice versa.

Definition 2.5. Let $f$ and $g$ be any two functions of $L^{1}(0, \infty)$. Then

$$
f \# g(x)=\int_{0}^{\infty} f(x, y) g(y) d \mu(y), \quad 0<x<\infty .
$$

We find that the properties of the \#-convolution parallel those of the *-convolution, as illustrated by the following lemmas.

LEMMA 2.6. Let $f$ and $g$ be functions of $L^{1}(0, \infty)$ and let

$$
f \# g(x)=\int_{0}^{\infty} f(x, y) g(y) d \mu(y), \quad 0<x<\infty .
$$

Then the integral defining $f$ \# $g(x)$ converges for almost all $x, 0<x<\infty$, and

$$
\|f \# g\|_{1} \leqq\|f\|_{1}\|g\|_{1} \text {. }
$$

Proof. We have

$$
\begin{aligned}
\int_{0}^{\infty}|f \# g(x)| d_{\mu}(x) & =\int_{0}^{\infty} d \mu(x)\left|\int_{0}^{\infty} f(x, t) g(t) d_{\mu}(t)\right| \\
& =\int_{0}^{\infty} d \mu(x)\left|\int_{0}^{\infty} g(t) d \mu(t) \int_{0}^{\infty} f(u) D(x, t, u) d \mu(u)\right| \\
& \leqq \int_{0}^{\infty} d \mu(x) \int_{0}^{\infty}|g(t)| d \mu(t) \int_{0}^{\infty}|f(u)| D(x, t, u) d \mu(u) \\
& =\int_{0}^{\infty}|g(t)| d_{\mu}(t) \int_{0}^{\infty}|f(u)| d_{\mu}(u) \int_{0}^{\infty} D(x, t, u) d \mu(x) .
\end{aligned}
$$

The change in order of integration follows by Fubini's theorem. It is thus clear that

$$
\|f \# g\|_{1} \leqq\|f\|_{1}\|g\|_{1} .
$$

LEммa 2.7. If $f$ and $g$ are functions of $L^{1}(0, \infty)$, then 


$$
(f \# g) \wedge(x)=f^{\wedge}(x) g^{\wedge}(x), \quad 0<x<\infty .
$$

Proof. We have

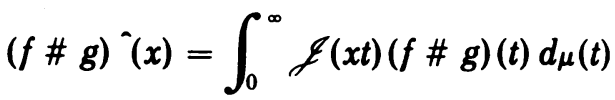

$$
\begin{aligned}
& =\int_{0}^{\infty} \not{f}(x t) d \mu(t) \int_{0}^{\infty} f(t, u) g(u) d \mu(u) \\
& =\int_{0}^{\infty} f(x t) d \mu(t) \int_{0}^{\infty} g(u) d \mu(u) \int_{0}^{\infty} f(v) D(t, u, v) d \mu(v) \\
& =\int_{0}^{\infty} g(u) d \mu(u) \int_{0}^{\infty} f(v) d \mu(v) \int_{0}^{\infty} \mathscr{f}(x t) D(t, u, v) d \mu(t),
\end{aligned}
$$

the change in order of integration follows by Fubini's theorem. Thus, by $\$ 1,(5)$, we have

$$
\begin{aligned}
(f \# g) \stackrel{(}{(x)} & =\int_{0}^{\infty} \mathscr{f}(x u) g(u) d_{\mu}(u) \int_{0}^{\infty} \mathscr{f}(x v) f(v) d_{\mu}(v) \\
& =f^{\wedge}(x) g^{\wedge}(x) .
\end{aligned}
$$

The following lemma may be found in $[5$, p. 315$]$. It enables us to establish the commutativity and associativity of the \#-convolution in $L^{1}(0, \infty)$.

LemMa 2.8. Let $f$ be a function of $L^{1}(0, \infty)$. Then

$$
f(x)=\lim _{n \rightarrow \infty} \int_{0}^{\infty} f^{-}(t) \hat{k_{n}^{n}}(t) \mathscr{J}(x t) d \mu(t) \quad \text { a.e. }
$$

where the $k_{n}(t), n=0,1,2, \cdots$, are a sequence of functions such that

(i) $k_{n}(t) \geqq 0,0<x<\infty$;

(ii) $\int_{0}^{\infty} k_{n}(t) d \mu(t)=1$,

(iii) $\lim _{n \rightarrow \infty} \int_{\delta}^{\infty} k_{n}(t) d_{\mu}(t)=0$ for every $\delta>0$,

(iv) $\hat{k_{n}}(t) \in L^{1}(0, \infty)$,

(v) $k_{n}^{n-}(x)=k_{n}(x)$.

An example of a sequence of functions $k_{n}$ satisfying the above properties is given by

$$
k_{n}(x)=\frac{1}{\Gamma(2 \gamma+1)} 2^{\gamma-1 / 2} \Gamma(\gamma+1 / 2) n^{2 \gamma+1} e^{-n x} .
$$

COROLLARY 2.9. Let $f$ and $g$ be functions of $L^{1}(0, \infty)$ such that $f^{\wedge}(x)=g^{\wedge}(x)$, $0<x<\infty$. Then $f(t)=g(t)$ a.e.

Corollary 2.10. Let $f$ be such that $f$ and $f^{\wedge}$ belong to $L^{1}(0, \infty)$. Then $\left(f^{\prime}\right)(x)=f(x)$ a.e.

Lemмa 2.11. Let $f, g$ and $h$ all be functions of $L^{1}(0, \infty)$. Then $(f \# g)(x)$ 
$=(g \# f)(x)$ a.e. and $((f \# g) \# h)(x)=(f \#(g \# h))(x)$ a.e.

Proof. We have $(f \# g) \wedge(x)=f^{\wedge}(x) g^{\wedge}(x)=g^{\wedge}(x) f^{\wedge}(x)=(g \# f)^{\wedge}(x)$ and hence, by Corollary 2.9, $(f \# g)(x)=(g \# f)(x)$ a.e. and similarly for associativity.

We conclude the section by including, without proof, the following general theorem on the application of linear differential operators to integrals.

THEOREM 2.12. Let $r_{m}(x), m=0,1, \cdots, M$ be functions continuous in $(a, b)$, $r_{M}(x)>0$. Define the linear differential operator

$$
L_{M}=\sum_{m=0}^{M} r_{m}(x)(d / d x)^{m}
$$

If $f(x)=\int_{0}^{\infty} Q(x, y) \phi(y) d \mu(y)$ converges for $0 \leqq x<\infty$, and if $q(x)$ $=\int_{0}^{\infty} L_{M}[Q(x, y)]_{\phi}(y) d_{\mu}(y)$ converges uniformly for $0 \leqq x \leqq A<\infty$ for every real number $A$, then $L_{M}[f(x)]=q(x)$.

\section{Chapter III. Variation diminishing \# -Kernels}

1. Introduction. In this chapter, we study properties of the variation diminishing \#-kernels $G(x)$ and of their associated functions $G(x, y)$ defined by $G(x)=\int_{0}^{\infty}(\mathscr{f}(x t) / E(t)) d_{\mu}(t)$ and

$$
G(x, y)=\int_{0}^{\infty} G(u) D(x, y, u) d_{\mu}(u) .
$$

We propose, first, to establish inequalities on $E(x)$ in order to verify the existence of basic integrals. We then construct the variation diminishing kernels $G(x)$ as convolutions of elementary, basic variation diminishing kernels. We examine variation diminishing matrices and explore properties of the associated functions $G(x, y)$.

2. Inequalities for $E(s)$.

LEMMA 2.1. Let $0<a_{1} \leqq a_{2} \leqq \cdots<\infty$, with $\sum_{k=1}^{\infty}\left(1 / a_{k}^{2}\right)<\infty$, and let $E(s)$ $=\coprod \coprod_{k=1}^{\infty}\left[1+s^{2} / a_{k}^{2}\right]$. Then $|E(\sigma+i \tau)| \geqq|E(i \tau)|$.

The proof follows immediately from the product representation of $E(s)$. Note that if $a_{k}=\infty$ for $k=n+1, n+2, \cdots$, then $E(s)$ becomes a polynomial of degree $2 n$, but the result holds nonetheless.

Theorem 2.2. Let $E(s)$ be the function defined in the preceding lemma. Then, for any positive numbers $p$ and $R .1 /\left|E\left(\sigma+i_{\tau}\right)\right|=O\left(1 /|\sigma|^{p}\right),|\sigma| \rightarrow \infty$, uniformly for $|\tau| \leqq R$.

The proof follows from the definition of $E(s)$ and the preceding lemma. Note that if $E(s)$ is a polynomial of degree $2 n$, the theorem holds for $p \leqq 2 n$.

The following results will all be based on the assumption that $E(s)$ is an infinite product. 
3. Generation of variation diminishing kernels. We propose to show that the kernels $G(x)$ referred to in the Introduction may be generated from an infinite number of \# -convolutions of elementary basic variation diminishing kernels $g_{a}(x), a>0$, given by

$$
g_{a}(x)=a^{2 \gamma+1} \mathscr{K}(a x),
$$

where

$$
\mathscr{K}^{\prime}(x)=x^{1 / 2-\gamma} K_{\gamma-1 / 2}(x),
$$

$K_{\gamma-1 / 2}(x)$ being the Bessel function of the second kind. In our development we also need the function

$$
\mathscr{I}(x)=2^{\gamma-1 / 2} \Gamma(\gamma+1 / 2) x^{1 / 2-\gamma} I_{\gamma-1 / 2}(x),
$$

where $I_{\gamma-1 / 2}(x)$ is the Bessel function of imaginary argument of order $\gamma-1 / 2$.

Lemma 3.1. Let $g_{a}(x)$ be defined by (1). Then $g_{a}(x)=1 /\left(1+x^{2} / a^{2}\right)$.

Proof. See [17, p. 410].

Lemma 3.2. Let $\mathscr{P}(a x)$ and $\mathscr{K}(a x)$ be defined by (3) and (2) above. Then

$$
\begin{aligned}
\left(1-\frac{\Delta_{x}}{a^{2}}\right) f(x) & =-\frac{1}{a^{2} x^{2 \gamma} \mathscr{Y}(a x)} \frac{d}{d x}\left[x^{2 \gamma}(\mathscr{I}(a x))^{2} \frac{d}{d x} \frac{f(x)}{\mathscr{I}(a x)}\right] \\
& =-\frac{1}{a^{2} x^{2 \gamma} \mathscr{K}(a x)} \frac{d}{d x}\left[x^{2 \gamma}(\mathscr{K}(a x))^{2} \frac{d}{d x} \frac{f(x)}{\mathscr{K}(a x)}\right] .
\end{aligned}
$$

Proof. Each of the identities may be verified by straight computation of the right-hand side, making use of Bessel's equation.

LEMma 3.3.

$$
\mathscr{K}(a x) \frac{d}{d x} \mathscr{P}(a x)-\mathscr{I}(a x) \frac{d}{d x} \mathscr{K}(a x)=\frac{2^{\gamma-1 / 2} \Gamma(\gamma+1 / 2)}{x^{2 \gamma} a^{2 \gamma-1}} .
$$

Proof. See [17, pp. 79, 80].

LEMMA 3.4. Let

$$
g_{a}(x, y)=\int_{0}^{\infty} \frac{f(x t) \not f(y t)}{1+\frac{t^{2}}{a^{2}}} d \mu(t)
$$

Then

$$
\begin{aligned}
g_{a}(x, y) & =a^{2 \gamma+1} \mathscr{K}(a y) \mathscr{P}(a x), & & x \leqq y, \\
& =a^{2 \gamma+1} \mathscr{K}(a x) \mathscr{P}(a y), & & x \geqq y .
\end{aligned}
$$

Proof. See [17, p. 429]. 
Lemma 3.5. Let $g_{a}(x, y)$ be the function defined in Lemma 3.4 and let $\phi(x)$ be a continuous function of $L^{\infty}(0, \infty)$. Then

$$
\left(1-\frac{\Delta_{x}}{a^{2}}\right) \int_{0}^{\infty} g_{a}(x, y) \phi(y) d \mu(y)=\phi(x) .
$$

Proof. By Lemma 3.4, we have

$$
\begin{aligned}
\int_{0}^{\infty} g_{a}(x, y) \phi(y) d \mu(y) & =\int_{0}^{x} g_{a}(x, y) \phi(y) d \mu(y)+\int_{x}^{\infty} g_{a}(x, y) \phi(y) d \mu(y) \\
= & a^{2 \gamma+1}\left[\mathscr{K}(a x) \int_{0}^{x} \mathscr{P}(a y) \phi(y) d \mu(y)\right. \\
& \left.+\mathscr{P}(a x) \int_{x}^{\infty} \mathscr{K}(a y) \phi(y) d \mu(y)\right] .
\end{aligned}
$$

Applying the operator $\left(1-\Delta_{x} / a^{2}\right)$ to each side and using Leibniz's rule, we have, because of Bessel's equations,

$$
\begin{aligned}
(1 & \left.-\frac{\Delta_{x}}{a^{2}}\right) \int_{0}^{\infty} g_{a}(x, y) \phi(y) d \mu(y) \\
& =a^{2 \gamma+1}\left[-\frac{\phi(x)}{a^{2}} \frac{2^{1 / 2-\gamma} x^{2 \gamma}}{\Gamma\left(\gamma+\frac{1}{2}\right)}\right]\left[\mathscr{I}(a x) \frac{d}{d x} \mathscr{K}(a x)-\mathscr{K}(a x) \frac{d}{d x} \mathscr{P}(a x)\right] .
\end{aligned}
$$

Hence the result follows by Lemma 3.3.

TheOREM 3.6. $g_{a}(x), a>0$, is a variation diminishing kernel.

Proof. See [5, p. 322].

Lemma 3.7. Let $g_{1}(x), g_{2}(x), \ldots, g_{n}(x)$ be a sequence of variation diminishing kernels. Then, if

$$
g(x)=\left(g_{1} \# g_{2} \# \ldots \# g_{n}\right)(x),
$$

$g(x)$ is variation diminishing.

Proof. See [5, p. 323].

TheOREM 3.8. Let

$$
E(x)=\prod_{k=1}^{\infty}\left(1+\frac{x^{2}}{a_{k}^{2}}\right),
$$

where $0<a_{1} \leqq a_{2} \leqq \cdots$ and $\sum_{k=1}^{\infty}\left(1 / a_{k}^{2}\right)<\infty$, and let

$$
G(t)=\int_{0}^{\infty} \frac{\not f(x t)}{E(x)} d \mu(x) \text {. }
$$

Then 


$$
G^{\prime}(x)=\int_{0}^{\infty} \mathcal{f}(x t) G(t) d \mu(t)=\frac{1}{E(x)},
$$

and $G(t)$ is a variation diminishing \#-kernel.

Proof. See [5, p. 323].

\section{Matrix transformations.}

Definition 4.1. A real matrix $A=\left|a_{i j}\right|(1 \leqq i \leqq m ; 1 \leqq j \leqq n)$ is called totally non-negative if all its minors of any order are non-negative.

DEFINITION 4.2. Let $A=\left\lfloor a_{i j}\right\rfloor(1 \leqq i \leqq m ; 1 \leqq j \leqq n)$ be a real matrix of order $R$. Then $A$ is called minor definite if all the following of its minors have the same sign:

(a) those which are of equal order $r<R$;

(b) those which are of order $=R$ and which belong to the same combination of columns.

Definition 4.3. A real matrix $A=\left[a_{i j}\right](1 \leqq i \leqq m ; 1 \leqq j \leqq n)$ is said to be variation diminishing if and only if for any set of real numbers $x_{1}, \cdots, x_{n}$, it follows that

$$
y_{i}=\sum_{j=1}^{n} a_{i j} x_{j}, \quad i=1, \cdots, m,
$$

implies that

$$
V\left[y_{1}, \cdots, y_{m}\right] \leqq V\left[x_{1}, \cdots, x_{n}\right] .
$$

The associated function of a variation diminishing \#-kernel will be referred to as variation diminishing. An approximation argument readily shows that if $G(x, y)$ is variation diminishing, so is the matrix $\left[G\left(x_{i}, y_{j}\right)\right]$ $(1 \leqq i \leqq n, 1 \leqq j \leqq m)$.

TheOREM 4.4. $\left[G\left(x_{i}, y_{j}\right)\right]_{1 \leqq i, j \leqq n}$ is totally non-negative.

Proof. Consider the quadratic form

$$
\begin{aligned}
\sum_{i, j=1}^{n} G\left(x_{i}, x_{j}\right) t_{i} t_{j} & =\sum_{i, j=1}^{n} t_{i} t_{j} \int_{0}^{\infty} \frac{\mathcal{f}\left(x_{i} u\right) \mathscr{f}\left(x_{j} u\right)}{E(u)} d_{\mu}(u) \\
& =\int_{0}^{\infty}\left[\sum_{i, j=1}^{n} t_{i} t_{j} \mathscr{f}\left(x_{i} u\right) \mathscr{f}\left(x_{j} u\right)\right] \frac{1}{E(u)} d_{\mu}(u) \\
& =\int_{0}^{\infty}\left[\sum_{i=1}^{n} \mathscr{f}\left(x_{i} u\right) t_{i}\right]^{2} \frac{1}{E(u)} d_{\mu}(u) \\
& \geqq 0 .
\end{aligned}
$$

By a theorem on quadratic forms (see $[2$, Vol. I, p. 306]), it follows that 
$\operatorname{det}\left[G\left(x_{i}, x_{j}\right)\right]_{i, j=1, \cdots, n}>0$. Now let $0 \leqq x_{1}<x_{2}<\cdots<x_{n}<\infty$ and $0 \leqq y_{1}<y_{2}$ $<\cdots<y_{n}<\infty$, and consider the set consisting of all the $x_{i}^{\prime}$ 's and $y_{i}^{\prime}$ 's. Arrange these in increasing order, denoting the numbers of the combined set by $w_{j}, j=1, \cdots, r$, where $0 \leqq w_{1}<w_{2}<\cdots<w_{r}$, with $n \leqq r \leqq 2 n$. Consider the $n \times r$ matrix $A=\left[G\left(x_{i}, w_{j}\right)\right](i=1, \cdots, n ; j=1, \cdots, r)$. By the remark preceding the theorem, $A$ is a variation diminishing matrix and hence, by $[10$, p. 101] $A$ is minor definite. Since $A$ has rank $n$, all $n \times n$ minors have the same sign, and since $\operatorname{det}\left[G\left(x_{i}, x_{j}\right)\right]_{i, j=1, \cdots, n} \geqq 0$, we also have $\operatorname{det}\left[G\left(x_{i}, y_{j}\right)\right]_{i, j=1, \cdots, n} \geqq 0$.

TheOREM 4.5. $G(x, y)>0$.

Proof. By the preceding theorem, with $n=1$, it is clear that $G(x, y) \geqq 0$. Suppose $G(x, y)=0$. Then we may appeal to IV, Theorem 3.2 (the proof of which, though independent of the preceding results, is more conveniently included in IV) to note that there exists a number $y_{1}>y$ such that $G\left(x, y_{1}\right)$ $>0$. Similarly, since $G(x, y)=G(y, x)$, there exists a number $x_{1}>x$ such that $G\left(x_{1}, y\right)>0$. Now, applying the preceding theorem once more, with $n=2$, we find that

$$
\left|\begin{array}{cc}
G(x, y) & G\left(x, y_{1}\right) \\
G\left(x_{1}, y\right) & G\left(x_{1}, y_{1}\right)
\end{array}\right| \geqq 0
$$

or

$$
-G\left(x, y_{1}\right) G\left(x_{1}, y\right) \geqq 0
$$

a contradiction.

5. Properties of $G_{N}(x, y)$. Let

$$
E_{N}(x)=\prod_{k=N+1}^{\infty}\left(1+\frac{x^{2}}{a_{k}^{2}}\right),
$$

where $0<a_{1} \leqq a_{2} \leqq \cdots$, with $\sum_{k=N+1}^{\infty}\left(1 / a_{k}^{2}\right)<\infty$, and let $G_{N}(x)$ be the variation diminishing kernel whose Hankel transform is the reciprocal of $E_{N}(x)$, and $G_{N}(x, y)$ be the function associated with $G_{N}(x)$. We write $G(x)$ and $G(x, y)$ for $G_{0}(x)$ and $G_{0}(x, y)$, respectively.

The following theorems will be stated for $G(x, y)$, though they hold equally well for $G_{N}(x, y)$ with $N$ any integer.

Theorem 5.1. If $G(x, y)$ is the function associated with $G(x)$, then

$$
G(x, y)=\int_{0}^{\infty} \frac{f(x t) \mathscr{f}(y t)}{E(t)} d \mu(t) .
$$

Proof. By definition, we have 


$$
\begin{aligned}
G(x, y) & =\int_{0}^{\infty} G(u) D(x, y, u) d_{\mu}(u) \\
& =\int_{0}^{\infty} D(x, y, u) d_{\mu}(u) \int_{0}^{\infty} \frac{\mathcal{f}(t u)}{E(t)} d_{\mu}(t) \\
& =\int_{0}^{\infty} \frac{1}{E(t)} d_{\mu}(t) \int_{0}^{\infty} \mathcal{f}(t u) D(x, y, u) d_{\mu}(u),
\end{aligned}
$$

where the change in order of integration follows by Fubini's theorem. Applying II, \$1, (5), we have the required result.

Note that $G(x, 0)=G(x)$.

Corollary 5.2. $\left(\partial^{m+n} / \partial x^{m} \partial y^{n}\right) G(x, y), m, n=0,1,2, \cdots$, is bounded and continuous for $0 \leqq x, y<\infty$.

TheOREM 5.3. $\int_{0}^{\infty} G(x, y) d \mu(y)=1,0 \leqq x<\infty$.

Proof. We have

$$
\begin{aligned}
\int_{0}^{\infty} G(x, y) d_{\mu}(y) & =\int_{0}^{\infty} d_{\mu}(y) \int_{0}^{\infty} G(t) D(x, y, t) d_{\mu}(t) \\
& =\int_{0}^{\infty} G(t) d_{\mu}(t) \int_{0}^{\infty} D(x, y, t) d_{\mu}(y) \\
& =\int_{0}^{\infty} G(t) d_{\mu}(t)=1
\end{aligned}
$$

THEOREM 5.4. Let $G(x, y)$ be the function associated with $G(x)$. Then, for $x$ fixed,

(a) $\int_{0}^{\infty} t^{2} G(x, t) d_{\mu}(t)=x^{2}+2(2 \gamma+1) A_{2}$

(b) $\int_{0}^{\infty} t^{4} G(x, t) d_{\mu}(t)=x^{4}+4 x^{2}(2 \gamma+3) A_{2}+8(2 \gamma+1)(2 \gamma+3)\left(A_{2}^{2}-A_{4}\right)$,

(c) $\int_{0}^{\infty}\left(t^{2}-x^{2}\right)^{2} G(x, t) d \mu(t)=8 x^{2} A_{2}+4(2 \gamma+1)(2 \gamma+3)\left(A_{2}^{2}-A_{4}\right)$, where $A_{2}=\sum_{k=1}^{\infty}\left(1 / a_{k}^{2}\right), A_{4}=\sum_{1 \leqq i<j}^{\infty}\left(1 / a_{i}^{2} a_{j}^{2}\right)$.

Proof. We have

$$
\int_{0}^{\infty} G(x, t) \not{f}(y t) d_{\mu}(t)=\frac{\mathscr{J}(x y)}{E(y)} .
$$

The definitions of $f(x y)$ and $E(y)$ give us the following Taylor expansions:

$$
\begin{aligned}
& f(x y)=1-\frac{x^{2}}{2(2 \gamma+1)} y^{2}+\frac{x^{4}}{8(2 \gamma+1)(2 \gamma+3)} y^{4}-\cdots \\
& E(y)=1+A_{2} y^{2}+A_{4} y^{4}+\cdots
\end{aligned}
$$

Hence 
(4)

$$
\begin{aligned}
\frac{\mathscr{J}(x y)}{E(y)}=1 & -\left[\frac{x^{2}}{2(2 \gamma+1)}+A_{2}\right] y^{2} \\
& +\left[\frac{x^{4}}{8(2 \gamma+1)(2 \gamma+3)}+\frac{x^{2}}{2(2 \gamma+1)} A_{2}+A_{2}^{2}-A_{4}\right] y^{4}+\cdots
\end{aligned}
$$

Substituting (2) and (4) in (1), we find that

$$
\begin{aligned}
\int_{0}^{\infty} G(x, t) & {\left[1-\frac{t^{2}}{2(2 \gamma+1)} y^{2}+\frac{t^{4}}{8(2 \gamma+1)(2 \gamma+3)} y^{4}-\cdots\right] d \mu(t) } \\
=1 & -\left[\frac{x^{2}}{2(2 \gamma+1)}+A_{2}\right] y^{2} \\
+ & {\left[\frac{x^{4}}{8(2 \gamma+1)(2 \gamma+3)}+\frac{x^{2}}{2(2 \gamma+1)} A_{2}+A_{2}^{2}-A_{4}\right] y^{4}+\cdots }
\end{aligned}
$$

Equating coefficients of $y^{2}$ and $y^{4}$, we obtain (a) and (b), respectively, whereas (c) is a direct result of (a), (b) and Theorem 5.3.

Corollary 5.5.

$$
\int_{0}^{\infty} t^{2} G_{N}(t) d \mu(t)=2(2 \gamma+1) \sum_{k=N+1}^{\infty}\left(\frac{1}{a_{k}^{2}}\right) .
$$

Corollary 5.6. For any $\delta>0$,

$$
\lim _{N \rightarrow \infty} \int_{\delta}^{\infty} G_{N}(t) d \mu(t)=0 .
$$

Proof. We have, for fixed $\delta>0$,

$$
\begin{aligned}
\int_{\delta}^{\infty} G_{N}(t) d \mu(t) & \leqq \frac{1}{\delta^{2}} \int_{\delta}^{\infty} t^{2} G_{N}(t) d \mu(t) \\
& =\frac{2(2 \gamma+1)}{\delta^{2}} \sum_{k=N+1}^{\infty} \frac{1}{a_{k}^{2}},
\end{aligned}
$$

and the result is immediate on letting $N \rightarrow \infty$.

Theorem 5.7. For $x$ fixed, $0<x<\infty$,

$$
\begin{aligned}
\lim _{N \rightarrow \infty} \int_{a}^{b} G_{N}(x, y) d \mu(y) & =1, & & 0 \leqq a<x<b \leqq \infty, \\
& =0, & & 0 \leqq a \leqq b<x<\infty, \\
& =0, & & 0<x<a \leqq b \leqq \infty .
\end{aligned}
$$

Proof. Suppose $0 \leqq a \leqq b<x<\infty$. Let $b=x-\delta, \delta>0$. Then we have 


$$
\begin{aligned}
\int_{a}^{b} G_{N}(x, y) d \mu(y) & \leqq \int_{0}^{x-\delta} G_{N}(x, y) d \mu(y) \\
& =\int_{0}^{x-\delta} d \mu(y) \int_{0}^{\infty} G_{N}(u) D(x, y, u) d \mu(u) \\
& \leqq \int_{0}^{\infty} d \mu(y) \int_{\delta}^{\infty} G_{N}(u) D(x, y, u) d \mu(u) \\
& =\int_{\delta}^{\infty} G_{N}(u) d \mu(u) \int_{0}^{\infty} D(x, y, u) d \mu(y) \\
& =\int_{\delta}^{\infty} G_{N}(u) d \mu(u) .
\end{aligned}
$$

The result we seek follows directly on letting $N \rightarrow \infty$ and taking note of Corollary 5.6. The proof for $0<x<a \leqq b \leqq \infty$ is similar, and that for $0 \leqq a<x<b \leqq \infty$ follows from the first two cases and Theorem 5.3.

Let us define a frequency function as any non-negative function $\phi(t)$ for which $\int_{0}^{\infty} \phi(t) d_{\mu}(t)=1$. The mean, $m_{\phi}$, and the variance, $V_{\phi}$, of the frequency function $\phi$ are defined by

$$
\begin{aligned}
& m_{\phi}^{2}=\int_{0}^{\infty} t^{2} \phi(t) d \mu(t), \\
& V_{\phi}=\int_{0}^{\infty}\left(t^{2}-m_{\phi}^{2}\right)^{2} \phi(t) d \mu(t) .
\end{aligned}
$$

We note that the associated functions $G_{N}(x, y)$ are frequency functions, and, by Theorem 5.4 ,

$$
\begin{aligned}
& m_{G_{N}}^{2}=x^{2}+2(2 \gamma+1) \sum_{k=N+1}^{\infty} \frac{1}{a_{k}^{2}}, \\
& V_{G_{N}}=8 x^{2} \sum_{k=N+1}^{\infty} \frac{1}{a_{k}^{2}} \\
& \quad+4(2 \gamma+1)(2 \gamma+3)\left[\left(\sum_{k=N+1}^{\infty} \frac{1}{a_{k}^{2}}\right)^{2}-\sum_{N+1 \leqq i<j}^{\infty} \frac{1}{a_{i}^{2} a_{j}^{2}}\right],
\end{aligned}
$$

where $x$ is fixed. It follows that as $N \rightarrow \infty, m_{G_{N}} \rightarrow x$ and $V_{G_{N}} \rightarrow 0$.

Chapter IV. Convergence of the convolution transform

1. Introduction. In this chapter, we develop asymptotic estimates for the variation diminishing kernel and its associated function. This leads us to the establishment of the convergence behavior of the \#-convolution transform. We find, in fact, that $G \# \phi(x)$ converges for all $x>0$ if $G \# \phi\left(x_{0}\right)$ converges for some $x_{0}>0$. Hence, we may refer to $G \# \phi$ as convergent or 
divergent. In this respect, we note that $G \# \phi$ behaves like the Stieltjes transform.

\section{Residues.}

THEOREM 2.1. Let $z=a_{1} i$ be a zero of order $m_{1}+1$ of $E(z)$. Then the residue $R$ of

$$
\frac{z^{1 / 2+\gamma} H_{r-1 / 2}^{(1)}(z y) \not f(z x)}{E(z)}
$$

at $z=a_{1} i$ is

$$
\left[\left(\frac{d}{d a}\right)^{m_{1}}\left\{\psi(a) \mathscr{P}(a x) K_{\gamma-1 / 2}(a y)\right\}\right]_{a=a_{1}},
$$

where $\psi\left(a_{1}\right) \neq 0$.

The proof, involving only direct computation, is omitted.

Corollary 2.2. With the notation of the theorem, for $x$ fixed, $0 \leqq x<\infty$,

$$
R \sim C \mathscr{P}\left(a_{1} x\right) y^{m_{1}-1 / 2} e^{-a_{1} y}, \quad y \rightarrow \infty,
$$

where $C, a$ constant independent of $x$ and $y$, is positive.

Proof. By the theorem, we have

$$
R=\left[\left(\frac{d}{d a}\right)^{m_{1}}\left\{\psi(a) \mathscr{P}(a x) K_{\gamma-1 / 2}(a y)\right\}\right]_{a=a_{1}} .
$$

On carrying out the differentiation, we note that the dominant term in $y$, for fixed $x$, is of the form

$$
B \mathcal{P}(a x) y^{m_{1}} K_{\gamma-1,2-m_{1}}\left(a_{1} y\right), \quad B>0 .
$$

Since, by $[17$, p. 202$]$,

$$
K_{\gamma-1,2-m_{1}}\left(a_{1} y\right)=\left(\frac{\pi}{2 a_{1} y}\right)^{1 / 2} e^{-a_{1} y}\left[1+O\left(\frac{1}{y}\right)\right],
$$

the result follows.

3. Asymptotic estimate for $G(x, y)$.

Lemma 3.1.

$$
G(x, y)=y^{1:-} \int_{C} \frac{y(x t)}{E(t)} t^{\gamma+1}{ }^{2} H_{\curlyvee-12}^{(1)}(y t) d t,
$$

where $C$ is the contour

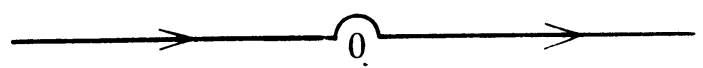


Proof. The definitions of $\mathscr{f}(z)$ and $d_{\mu}(z)$ give

$$
\mathscr{f}(y t) d_{\mu}(t)=y^{1 / 2-\gamma} t^{1 / 2+\gamma} J_{\gamma-1 / 2}(y t) d t,
$$

and since

$$
G(x, y)=\int_{0}^{\infty} \frac{\not{f}(x t) \not{f}(y t)}{E(t)} d \mu(t)
$$

we have

$$
G(x, y)=y^{1 / 2-\gamma} \int_{0}^{\infty} \frac{\mathscr{J}(x t)}{E(t)} t^{1 / 2+\gamma} J_{\gamma-1 / 2}(y t) d t .
$$

By $[17$, p. 75$]$,

$$
\begin{aligned}
G(x, y)= & y^{1 / 2-\gamma} \int_{0}^{\infty} \frac{\mathscr{J}(x t)}{E(t)} t^{1 / 2+\gamma}\left[H_{\gamma-1 / 2}^{(1)}(y t)-e^{(\gamma-1 / 2) \pi i} H_{\gamma-1 / 2}^{(1)}\left(y t e^{\pi i}\right)\right] d t \\
= & y^{1 / 2-\gamma}\left[\int_{0}^{\infty} \frac{t^{1 / 2+\gamma} \mathscr{f}(x t)}{E(t)} H_{\gamma-1 / 2}^{(1)}(y t) d t\right. \\
& \left.-e^{(\gamma-1 / 2) \pi i} \int_{0}^{-\infty} \frac{\left(t e^{-\pi i}\right)^{1 / 2+\gamma} \mathscr{f}\left(x t e^{-\pi i}\right) H_{\gamma-1 / 2}(y t) e^{-\pi i}}{E\left(t e^{-\pi i}\right)} d t\right] \\
= & y^{1 / 2-\gamma}\left[\int_{0}^{\infty} \frac{\mathscr{J}(x t) t^{1 / 2+\gamma} H_{\gamma-1 / 2}^{(1)}(y t)}{E(t)} d t+\int_{-\infty}^{0} \frac{\mathscr{J}(x t) t^{1 / 2+\gamma} H_{\gamma-1 / 2}^{(1)}(y t)}{E(t)} d t\right]
\end{aligned}
$$

since $\mathscr{Z}$ and $E$ are even functions.

TheOREM 3.2. Under the assumptions of Theorem 2.1, with $x$ fixed, $0 \leqq x<\infty$,

$$
G(x, y)=c e^{-a_{1} y} y^{m_{1}-\gamma} \mathscr{P}\left(a_{1} x\right)\left[1+O\left(\frac{1}{y}\right)\right], \quad y \rightarrow \infty,
$$

where $c>0$.

Proof. Let $a$ be such that $a_{1}<a<a_{2}$, and let $C$ be the indicated contour:

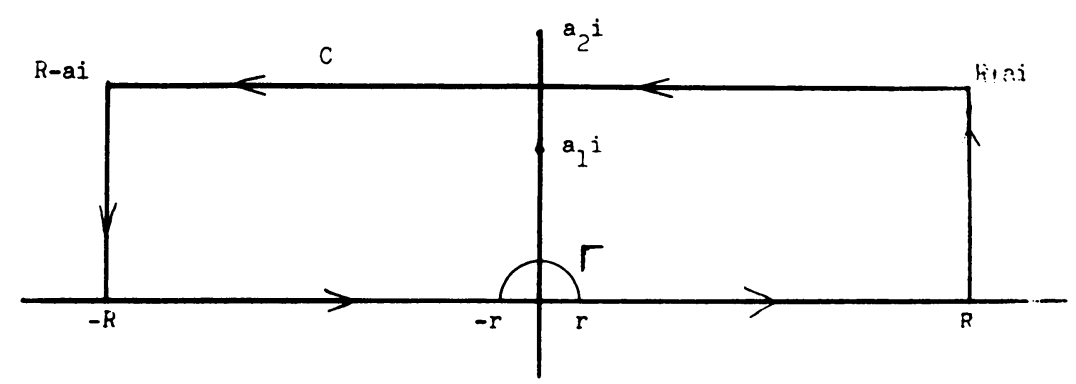

By the residue theorem, we have 


$$
\int_{C} \frac{z^{1 / 2+\gamma} \mathscr{J}(x z) H_{\gamma-1 / 2}^{(1)}(y z)}{E(z)} d z=2 \pi i\left\{\operatorname{Res} \frac{z^{1 / 2+\gamma} \mathscr{f}(x z) H_{\gamma-1 / 2}^{(1)}(y z)}{E(z)}\right\}_{z=a_{1} i}
$$

We may apply III, Theorem 2.2 and routine estimates of Bessel functions to get, on letting $R \rightarrow \infty$ and $r \rightarrow 0$,

$$
\int_{-\infty}^{\infty} \frac{t^{1,2+\gamma} \mathscr{f}(x t) H_{\gamma-1,2}^{(1)}(y t)}{E(t)} d t=2 \pi i\left\{\operatorname{Res} \frac{z^{1 / 2+\gamma} \mathscr{f}(x z) H_{\gamma-1 / 2}^{(1)}(y z)}{E(z)}\right\}_{z=q i}+S(x, y)
$$

where $|S(x, y)|=O\left(y^{-1 / 2} e^{-a y}\right), y \rightarrow \infty$. An appeal to Lemma 3.1 and Corollary 2.2 leads to the desired result.

\section{Convergence property.}

TheOREM 4.1. Let $\phi$ be a function integrable in every finite interval, and let

$$
\int_{0}^{\infty} G\left(x_{0}, y\right) \phi(y) d \mu(y)=\lim _{T \rightarrow \infty} \int_{0}^{T} G\left(x_{0}, y\right) \phi(y) d \mu(y), \quad x_{0} \geqq 0,
$$

converge conditionally. Then $\int_{0}^{\infty} G(x, y) \phi(y) d \mu(y)$ converges conditionally for all $x$ and uniformly for $x$ in any finite interval.

Proof. Let $0 \leqq s_{1}<s$ and $0 \leqq t_{1}<t$. Then, by III, Theorem 4.4, we have that

$$
\left|\begin{array}{cc}
G\left(s_{1}, t_{1}\right) & G\left(s_{1}, t\right) \\
G\left(s, t_{1}\right) & G(s, t)
\end{array}\right| \geqq 0
$$

or, by III, Theorem 4.5, $G(s, t) / G\left(s_{1}, t\right) \geqq G\left(s, t_{1}\right) / G\left(s_{1}, t_{1}\right)$. Let

$$
g(y)=\frac{G(x, y)}{G\left(x_{0}, y\right)}
$$

Then, clearly, $g(y)>0$, and if $x_{0}>x, g(y) \downarrow$. Hence

$$
0<g(y) \leqq g(0)=\frac{G(x, 0)}{G\left(x_{0}, 0\right)}=\frac{G(x)}{G\left(x_{0}\right)},
$$

and $g(y)$ is uniformly bounded for all $x<x_{0}$. On the other hand, if $x_{0}<x$,

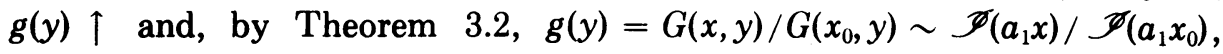
$y \rightarrow \infty$. Hence, in this case, $g(y)$ is uniformly bounded for all $x, x_{0} \leqq x \leqq A$, for every real number $A \geqq x_{0}$.

Now

$$
\begin{aligned}
\int_{0}^{\infty} G(x, y) \phi(y) d \mu(y) & =\int_{0}^{\infty} \frac{G(x, y)}{G\left(x_{0}, y\right)} G\left(x_{0}, y\right) \phi(y) d \mu(y) \\
& =\int_{0}^{\infty} g(y) G\left(x_{0}, y\right) \phi(y) d \mu(y)
\end{aligned}
$$


Using the second mean value theorem, we find that

$$
\begin{aligned}
& \left|\int_{T_{1}}^{T_{2}} G(x, y) \phi(y) d \mu(y)\right| \\
& \quad \leqq\left|g\left(T_{1}\right)\right|\left|\int_{T_{1}}^{w} G\left(x_{0}, y\right) \phi(y) d \mu(y)\right|+\left|g\left(T_{2}\right)\right|\left|\int_{\omega}^{T_{2}} G\left(x_{0}, y\right) \phi(y) d \mu(y)\right|,
\end{aligned}
$$

where $T_{1} \leqq w \leqq T_{2}$. Since $g(y)$ is uniformly bounded, and since, by hypothesis, we know that given $\epsilon>0$, there is a $T>0$ such that for $T_{1}, T_{2}>T$, each of the integrals on the right is less than $\epsilon$, the theorem follows.

Chapter V. INVERsion For CONTINUOUS FUnCtions

1. Introduction. In this chapter, we derive the inversion theorem under general hypotheses. Since the behavior of $G_{N}(x, y) / G(x, y)$ and of $G_{N}(x, y)$ plays a significant role in the development, we give a detailed study of the essential properties of these functions.

2. The changes of trend of $G_{N}(x, y) / G(x, y)$.

Lemma 2.1. Let $\Delta(s)=1-|s|$ for $|s|<1, \Delta s=0$, otherwise. Then

$$
G_{N}(x, y)=\lim _{h \rightarrow 0} \int_{0}^{\infty} G_{N}(s, y) \frac{\Gamma(\gamma+1 / 2) \Delta\left(\frac{x-s}{h}\right)}{2^{1 / 2-\gamma} s^{2 \gamma} h} d \mu(s) .
$$

Proof. We have

$$
\begin{aligned}
I & =\int_{0}^{\infty} G_{N}(s, y) \frac{\Gamma(\gamma+1 / 2) \Delta\left(\frac{x-s}{h}\right)}{2^{1 / 2-\gamma} s^{2 \gamma} h} d \mu(s) \\
& =\int_{x-h}^{x+h} G_{N}(s, y) \frac{\Delta\left(\frac{x-s}{h}\right)}{h} d s .
\end{aligned}
$$

Letting $s=x-s^{\prime} h$, we get

$$
I=\int_{-1}^{1} G_{N}\left(x-s^{\prime} h, y\right) \Delta\left(s^{\prime}\right) d s^{\prime} .
$$

Now

$$
\lim _{h \rightarrow 0} G_{N}\left(x-s^{\prime} h, y\right)=G_{N}(x, y),
$$

and

$$
\left|G_{N}\left(x-s^{\prime} h, y\right) \Delta\left(s^{\prime}\right)\right| \leqq\left\|G_{N}\right\|_{\alpha} .
$$

Hence, by Lebesgue's limit theorem, it follows that 


$$
\lim _{h \rightarrow 0} I=G_{N}(x, y) \int_{-1}^{1} \Delta\left(s^{\prime}\right) d s^{\prime}=G_{N}(x, y)
$$

LEMMA 2.2. Let

$$
E_{N}^{*}(t)=\frac{E(t)}{E_{N}(t)}=\coprod_{k=1}^{N}\left(1+\frac{t^{2}}{a_{k}^{2}}\right)
$$

and let

$$
G^{*}(t)=\int_{0}^{\infty} \frac{\mathscr{f}(t u)}{E_{N}^{*}(u)} d_{\mu}(u)
$$

Then

$$
G(x, y)=\int_{0}^{\infty} G_{N}(x, s) G_{N}^{*}(s, y) d_{\mu}(s)
$$

Proof. We have

$$
\int_{0}^{\infty} G_{N}(x, s) G_{N}^{*}(s, y) d \mu(s)=\left(G_{N} \# G_{N}^{*}(\cdot, y)\right)(x)
$$

But

$$
\begin{aligned}
\left(G_{N} \# G_{N}^{*}(\cdot, y)\right)^{-}(x) & =G_{N}^{\hat{N}}(x) G_{N}^{*}(\cdot, y)^{\wedge}(x) \\
& =\frac{1}{E_{N}(x)} \frac{\mathcal{J}(x y)}{E_{N}^{*}(x)}=\frac{\mathcal{J}(x y)}{E(x)}=G(\cdot, y)^{\wedge}(x) .
\end{aligned}
$$

The lemma thus follows by inversion.

Theorem 2.3. For any real number $a$, and $x$ fixed, $G(x, y)-a G_{N}(x, y)$ has at most two changes of sign for $0 \leqq y<\infty$.

Proof. By Lemmas 2.1 and 2.2, we have

$$
\begin{aligned}
& G(x, y)-a G_{N}(x, y) \\
& \quad=\lim _{h \rightarrow 0} \int_{0}^{\infty} G_{N}(y, s)\left[G_{N}^{*}(x, s)-\frac{a \Gamma(\gamma+1 / 2) \Delta\left(\frac{x-s}{h}\right)}{2^{1 / 2-\gamma} h s^{2 \gamma}}\right] d_{\mu}(s) .
\end{aligned}
$$

Since $G_{N}(x, y)$ is a variation diminishing kernel, it follows that, for $x$ fixed,

$$
\begin{aligned}
V\left[\int_{0}^{\infty} G_{N}(y, s)\left\{G_{N}^{*}(x, s)-\frac{a \Gamma(\gamma+1 / 2) \Delta\left(\frac{x-s}{h}\right)}{2^{1,2-\gamma} h s^{2 \gamma}}\right\} d \mu(s)\right] \\
\leqq V\left[G_{N}^{*}(x, y)-\frac{a \Gamma(\gamma+1 / 2) \Delta\left(\frac{x-y}{h}\right)}{2^{1,2-\gamma} h y^{2 \gamma}}\right],
\end{aligned}
$$


so that, by $[10$, p. 81$]$, for $x$ fixed,

$$
V\left[G(x, y)-\dot{a} G_{N}(x, y)\right] \leqq \liminf _{h \rightarrow 0^{+}} V\left[G_{N}^{*}(x, y)-\frac{a \Gamma(\gamma+1 / 2) \Delta\left(\frac{x-y}{h}\right)}{2^{1 / 2-\gamma} h y^{2 \gamma}}\right] .
$$

Since, for all sufficiently small $h$, and $x$ fixed,

$$
G_{N}^{*}(x, y)-\frac{a \Gamma(\gamma+1 / 2) \Delta\left(\frac{x-y}{h}\right)}{2^{1 / 2-\gamma} h y^{2 \gamma}}
$$

has at most two changes of sign, so does $G(x, y)-a G_{N}(x, y)$.

Theorem 2.4. For $x$ fixed, $\lim _{y \rightarrow \infty} G_{N}(x, y) / G(x, y)=0$.

Proof. Let $a_{1} i$ be a zero of order $m_{1}+1$ of $E(t)$. If $m_{1}+1>N$, then $a_{1} i$ is a zero of order $m_{1}^{\prime}=m_{1}+1-N$ of $E_{N}(t)$. Hence by IV, Theorem 3.2,

$$
G_{N}(x, y) \sim C_{N} \mathscr{\mathcal { Y }}\left(a_{1} x\right) e^{-a_{1} y} y^{m_{1}-N},
$$

so that $G_{N}(x, y) / G(x, y) \sim C_{N} y^{-N}$, and $\lim _{y \rightarrow \infty} G_{N}(x, y) / G(x, y)=0$. If $m_{1}+1$ $\leqq N$, then $a_{1} i$ is not a zero of $E_{N}(t)$, and, by the proof of IV, Theorem 3.2, it follows that

$$
G_{N}(x, y)=O\left(y^{-\gamma} e^{-a y}\right), \quad a>a_{1} .
$$

Thus, using IV, Theorem 3.2 for $G(x, y)$, we again find that

$$
\lim _{y \rightarrow \infty} \frac{G_{N}(x, y)}{G(x, y)}=0 .
$$

Theorem 2.5. For $x$ fixed, $G_{N}(x, y) / G(x, y)$ has at most one change of trend.

Proof. Consider, for some real number $a$,

$$
G(x, y)-a G_{N}(x, y)=a G(x, y)\left[\frac{1}{a}-\frac{G_{N}(x, y)}{G(x, y)}\right] .
$$

Since $G(x, y)>0$, it follows that

$$
V\left[G(x, y)-a G_{N}(x, y)\right]=V\left[\frac{1}{a}-\frac{G_{N}(x, y)}{G(x, y)}\right] .
$$

If $G_{N}(x, y) / G(x, y)$ were to have more than two changes of trend, there would, necessarily, exist a real number $a$ for which $V\left[G(x, y)-a G_{N}(x, y)\right]$ $>2$, contradicting Theorem 2.3. Suppose then that $G_{N}(x, y) / G(x, y)$ has two changes of trend. We have $G_{N}(x, y) / G(x, y)>0$. Suppose $G_{N}(x, y) / G(x, y)$ is increasing in the neighborhood of the origin as in Figure a. If the quotient had two changes of trend, it would have to decrease once and then increase for all large values of $y$, contradicting. Theorem 2.4. Hence in this case, 
$G_{N}(x, y) / G(x, y)$ must have exactly one change of trend. On the other hand, suppose the quotient is decreasing in the neighborhood of the origin as in Figure b. Then since $\lim _{y \rightarrow \infty}\left(G_{N}(x, y) / G(x, y)\right)=0$, if there were two changes of trend, there would exist a real number $a$ for which $V\left[G(x, y)-a G_{N}(x, y)\right]$ $=3$, contradicting Theorem 2.3. Hence in this case $G_{N}(x, y) / G(x, y)$ has no change in trend.

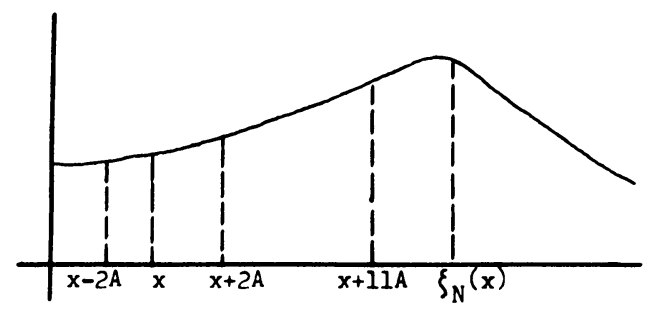

Figure a

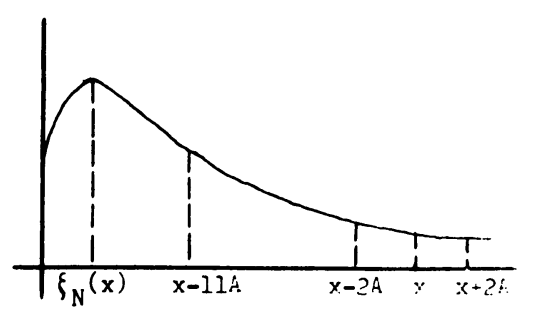

Figure b

Theorem 2.6. Let $x$ be fixed and $\delta$ any positive number. Then, for $N$ sufficiently large, $G_{N}(x, y) / G(x, y)$ has exactly one change of trend in the interval $(x-\delta, x+\delta)$.

Proof. Let $A$ be a number such that $0<A<1$. We note first that there must exist a $y$ in the interval $(x-\delta, x-\delta / 2)$ for which $G_{N}(x, y) / G(x, y)$ $<A$; for, otherwise, we have $G_{N}(x, y) / G(x, y) \geqq A$ for all $y, x-\delta<y<x$ $-\delta / 2$. By III, Theorem 5.7, it then follows that

$$
\begin{aligned}
0 & =\lim _{N \rightarrow \infty} \int_{x-\delta}^{x-\delta / 2} G_{N}(x, y) d \mu(y) \\
& =\lim _{N \rightarrow \infty} \int_{x-\delta}^{x-\delta / 2} \frac{G_{N}(x, y)}{G(x, y)} G(x, y) d \mu(y) \\
& \geqq A \int_{x-\delta}^{x-\delta, 2} G(x, y) d \mu(y)>0,
\end{aligned}
$$

a contradiction. Similarly, we may show that in the interval $(x+\delta / 2, x+\delta)$, there must exist a $y$ for which $G_{N}(x, y) / G(x, y)<A$. Finally, in the interval $(x-\delta / 2, x+\delta / 2)$, there must exist a $y$ for which $G_{N}(x, y) / G(x, y)>A$, for, otherwise, we have $G_{N}(x, y) / G(x, y) \leqq A$ for all $y$ in the interval. But then

$$
\begin{aligned}
\lim _{N \rightarrow \infty} \int_{x-\delta, 2}^{x+\delta, 2} G_{N}(x, y) d \mu(y) & =\lim _{N \rightarrow \infty} \int_{x-\delta, 2}^{x+\dot{2} 2} \frac{G_{N}(x, y)}{G(x, y)} G(x, y) d \mu(y) \\
& \leqq A \int_{x-\delta, 2}^{x+\delta, 2} G(x, y) d \mu(y) \leqq A<1
\end{aligned}
$$

On the other hand, III, Theorem 5.7, gives 


$$
\lim _{N \rightarrow \infty} \int_{x-\delta / 2}^{x+\delta, 2} G_{N}(x, y) d \mu(y)=1,
$$

a contradiction. This result in conjunction with Theorem 2.5, proves the theorem.

Theorem 2.7. For $x$ fixed and $\delta$ any positive number,

$$
\lim _{N \rightarrow \infty} \frac{G_{N}(x, y)}{G(x, y)}=0
$$

for $0 \leqq y \leqq x-\delta, x+\delta \leqq y \leqq \infty$.

Proof. By Theorem 2.6, we know that for $N$ sufficiently large, $G_{N}(x, y) / G(x, y)$ has one change of trend in the interval $(x-\delta / 2, x+\delta / 2)$. It therefore follows that $G_{N}(x, y) / G(x, y) \uparrow$ for $0 \leqq y \leqq x-\delta / 2$. Hence

$$
\int_{x-\delta}^{x-\delta, 2} \frac{G_{N}(x, y)}{G(x, y)} d \mu(y) \geqq \frac{G_{N}(x, x-\delta)}{G(x, x-\delta)} \int_{x-\delta}^{x-\delta, 2} d \mu(y) .
$$

Further, $G(x, y)>0$ so that $1 / G(x, y)$ is bounded. Hence

$$
\int_{x-\delta}^{x-\delta / 2} \frac{G_{N}(x, y)}{G(x, y)} d \mu(y) \leqq \underset{x-\delta \leqq y \leqq x-\delta / 2}{\text { l.u.b. }} \frac{1}{G(x, y)} \int_{x-\delta}^{x-\delta / 2} G_{N}(x, y) d_{\mu}(y) .
$$

Thus

$$
\frac{G_{N}(x, x-\delta)}{G(x, x-\delta)} \leqq{\underset{x}{x-\delta \leqq y \leqq x ~} \operatorname{u.b}_{j}}_{G(x, y)} \frac{\int_{x-\delta}^{x-\delta / 2} G_{N}(x, y) d \mu(y)}{\int_{x-\delta}^{x-\delta / 2} d_{\mu}(y)},
$$

and by III, Theorem 5.7, we have $\lim _{N \rightarrow \infty}\left(G_{N}(x, x-\delta) / G(x, x-\delta)\right)=0$. Similarly, we may show that $\lim _{N \rightarrow \infty}\left(G_{N}(x, x+\delta) / G(x, x+\delta)\right)=0$, and the theorem follows.

3. The change of trend of $G_{N}(x, y)$.

TheOREM 3.1. Let

$$
h_{t}(x, y)=\int_{0}^{\infty} \frac{\mathscr{f}(x t) \mathscr{J}(y t)}{e^{t^{2}, t^{2}}} d_{\mu}(t), \quad 0 \leqq x, y<\infty,
$$

and let

$$
G_{N, \mathrm{c}}(x, y)=\int_{0}^{\infty} G_{N}(s, y) h_{\mathrm{\epsilon}}(x, s) d \mu(s), \quad 0 \leqq x, y<\infty,
$$

Then

$$
\lim _{\rightarrow 00} \frac{G_{N, \mathrm{t}}(x, y)}{h_{\mathrm{t}}(x, x)}=1, \quad 0 \leqq x, y<\infty
$$


Proof. We have

$$
\begin{aligned}
h_{t}(x, y) & =\int_{0}^{\infty} \frac{f(x t) \mathscr{f}(y t)}{e^{t^{2}, \epsilon^{2}}} d \mu(t) \\
& =2^{\gamma-1,2} \Gamma(\gamma+1 / 2)(x y)^{1,2-\gamma} \int_{0}^{\infty} t e^{-t^{2}, \iota^{2}} J_{\gamma-1 / 2}(x t) J_{\gamma-1 / 2}(y t) d t,
\end{aligned}
$$

so that, by $[17$, p. 395$]$,

$$
h_{\mathrm{c}}(x, y)=\left(\frac{\epsilon^{2}}{2}\right)^{\gamma+1,2} e^{-\epsilon^{2}\left(x^{2}+y^{2}\right), 4} \not{P}\left(\frac{\epsilon^{2} x y}{2}\right) \text {. }
$$

Thus

$$
\frac{h_{\epsilon}(x, y)}{h_{\epsilon}(x, x)}=\frac{\mathcal{P}\left(\frac{\epsilon^{2} x y}{2}\right)}{\mathcal{P}\left(\frac{\epsilon^{2} x^{2}}{2}\right)} e^{-\epsilon^{2}\left(y^{2}-x^{2}\right), 4}
$$

and, since $\mathscr{P}(0)=1$, it follows that

$$
\lim _{c \rightarrow 0} \frac{h_{\mathrm{c}}(x, y)}{h_{\mathrm{c}}(x, x)}=1, \quad 0 \leqq x, y<\infty .
$$

Now we have

$$
\frac{G_{N, \mathrm{\epsilon}}(x, y)}{h_{\mathrm{\epsilon}}(x, x)}=\int_{0}^{\infty} G_{N}(s, y) \frac{h_{\mathrm{\epsilon}}(x, s)}{h_{\mathrm{\epsilon}}(x, x)} d_{\mu}(s) .
$$

It is clear that

$$
\lim _{t \rightarrow 0} G_{N}(s, y) \frac{h_{\epsilon}(x, s)}{h_{\epsilon}(x, x)}=G_{N}(s, y) .
$$

Further, for $0 \leqq x<\alpha$, the inequalities $1 \leqq \mathscr{F}(x) \leqq e^{x}$ readily follow from the power series expansion for $\mathscr{Y}(x)$. Hence

$$
\begin{aligned}
\left|\frac{h_{\epsilon}(x, s)}{h_{\epsilon}(x, x)}\right| & =\frac{\not{I}\left(\frac{\epsilon^{2} x s}{2}\right)}{\not\left(\frac{\epsilon^{2} x^{2}}{2}\right)} e^{-\epsilon^{2}\left(s^{2}-x^{2}\right), 4} \\
& \leqq e^{\epsilon^{2} x_{s} \cdot 2} e^{-\epsilon^{2}\left(s^{2}-x^{2}\right) / 4} \\
& =e^{x^{2} \cdot \epsilon^{2} \cdot e^{2}} e^{-\epsilon^{2}(s-x)^{2}+4}<e^{x^{2} \cdot 2},
\end{aligned}
$$

where the last inequality holds for all $s$ and for every $\epsilon<1$. Thus we may apply Lebesgue's limit theorem to get

$$
\lim _{t \rightarrow 0} \frac{G_{N, \mathrm{t}}(x, y)}{h_{\mathrm{t}}(x, x)}=\int_{0}^{\infty} G_{N}(s, y) d \mu(s)=1 .
$$


Theorem 3.2. For $x$ fixed and a any real number,

$$
V\left[G_{N}(x, y)-a\right] \leqq 2 .
$$

Proof. We have, by Lemma 2.1 , and by the definition of $G_{N, \epsilon}(x, y)$,

$$
\begin{aligned}
& G_{N}(x, y)-a G_{N, \mathrm{c}}(x, y) \\
& \quad=\lim _{h \rightarrow 0} \int_{0}^{\infty} G_{N}(s, y)\left[\frac{\Gamma(\gamma+1 / 2) \Delta\left(\frac{x-s}{h}\right)}{2^{1 / 2-\gamma} h s^{2 \gamma}}-a h_{\mathrm{c}}(x, s)\right] d_{\mu}(s),
\end{aligned}
$$

so that a proof entirely analogous to that of Theorem 2.3 , leads to the conclusion that

$$
V\left[G_{N}(x, y)-a G_{N, \mathrm{t}}(x, y)\right] \leqq 2 .
$$

Since $x$ is fixed and $a$ is arbitrary, we take $a=a^{\prime} / h_{\mathrm{t}}(x, x), a^{\prime}$ any real number. Then

$$
V\left[G_{N}(x, y)-a^{\prime} \frac{G_{N, \mathrm{e}}(x, y)}{h_{\mathrm{c}}(x, x)}\right] \leqq 2 .
$$

By Theorem 3.1 and $[10$, p. 84$]$, it follows that

$$
V\left[G_{N}(x, y)-a^{\prime}\right] \leqq \liminf _{\mathrm{c} \rightarrow 0} V\left[G_{N}(x, y)-a^{\prime} \frac{G_{N,}(x, y)}{h_{\mathrm{t}}(x, x)}\right] \leqq 2 .
$$

Theorem 3.3. For $x$ fixed, $\lim _{y \rightarrow \infty} G_{N}(x, y)=0$.

Proof. The conclusion is a direct result of IV, Theorem 3.2.

Theorem 3.4. For $x$ fixed, and for any $\delta>0, G_{N}(x, y)$ has a variation of trend in $(x-\delta, x+\delta)$ for $N$ sufficiently large.

Proof. We proceed in a manner entirely similar to the proof of Theorem 2.6. Let $A$ be a positive number such that $0<A \int_{x=\delta / 2}^{x+\delta / 2} d \mu(y)<1$. Within the interval $(x-\delta, x-\delta / 2)$, if $N$ is large enough, there must exist a $y$ for which $G_{N}(x, y)<A$; for, if not, $G_{n}(x, y) \geqq A$ for all $y$ in the interval. But then, applying III, Theorem 5.7, we find that

$$
\begin{aligned}
0 & =\lim _{N \rightarrow \infty} \int_{0}^{x-\delta, 2} G_{N}(x, y) d \mu(y) \\
& =\lim _{N \rightarrow \infty} \int_{0}^{x-\delta} G_{N}(x, y) d \mu(y)+\lim _{N \rightarrow \infty} \int_{x-\delta}^{x-\delta, 2} G_{N}(x, y) d \mu(y) \\
& =\lim _{N \rightarrow \infty} \int_{x-\delta}^{x-\delta, 2} G_{N}(x, y) d \mu(y) \\
& \geqq A \int_{x-\delta}^{x-\delta 2} d \mu(y)>0,
\end{aligned}
$$


a contradiction. Similarly, we may show that in the interval $(x+\delta / 2, x+\delta)$, there must exist a $y$ for which $G_{N}(x, y)<A$.

Moreover, in the interval $(x-\delta / 2, x+\delta / 2), G_{N}(x, y)$ must exceed $A$ at some point $y$; for, otherwise, we have $G_{N}(x, y) \leqq A$ for all $y$ in the interval. But then

$$
\lim _{N \rightarrow \infty} \int_{x-\delta, 2}^{x+\delta, 2} G_{N}(x, y) d \mu(y) \leqq A \int_{x-\delta, 2}^{x+\delta, 2} d_{\mu}(y)<1,
$$

by the definition of $A$; whereas, using III, Theorem 5.7 , we have

$$
\lim _{N \rightarrow \infty} \int_{x-\delta, 2}^{x+\delta, 2} G_{N}(x, y) d \mu(y)=1,
$$

a contradiction. It thus follows that for large $N, G_{N}(x, y)$ has a change of trend in the interval $(x-\delta, x+\delta)$.

Theorem 3.5. For $x$ fixed and $N$ sufficiently large, $G_{N}(x, y)$ has exactly one change of trend at $\xi_{N}(x), 0<\xi_{N}<\infty$, and $\left|x-\xi_{N}(x)\right| \leqq c\left(s_{N}\right)^{1 / 2}$, where $c$ is a constant depending only on $\gamma$, and $s_{N}=\sum_{k=N+1}^{a}\left(1 / a_{k}^{2}\right)$.

Proof. That $G_{N}(x, y)$ has at least one change of trend for $N$ sufficiently large is proved in Theorem 3.4. That $G_{N}(x, y)$ has at most one change of trend follows from the proof of Theorem 2.5 on replacing $G(x, y)$ by 1 . Thus it follows that for large $N, G_{N}(x, y)$ has exactly one change of trend at some point $\xi_{N}(x)$, and it remains for us to establish the bound for its distance from $x$. To this end, let

$$
A=\left(2(2 \gamma+1) s_{N}\right)^{1 / 2},
$$

and consider

(2) $\int_{x-2 A}^{x+2 A} G_{N}(x, y) d \mu(y)=1-\int_{0}^{x-2 A} G_{N}(x, y) d_{\mu}(y)-\int_{x+2 A}^{\infty} G_{N}(x, y) d \mu(y)$.

Now

$$
\begin{aligned}
\int_{0}^{x-2 A} G_{N}(x, y) d \mu(y) & =\int_{0}^{x-2 A} d \mu(y) \int_{0}^{\infty} G_{N}(u) D(x, y, u) d \mu(u) \\
& \leqq \int_{0}^{x-2 A} d \mu(y) \int_{2 A}^{2 x-2 A} G_{N}(u) D(x, y, u) d_{\mu}(u) \\
& \leqq \int_{2 A}^{2 x-2 A} G_{N}(u) d_{\mu}(u) \int_{0}^{\infty} D(x, y, u) d_{\mu}(y) \\
& \leqq \frac{1}{4 A^{2}} \int_{2 A}^{2 x-2 A} u^{2} G_{N}(u) d_{\mu}(u) \leqq \frac{1}{4},
\end{aligned}
$$

where the change in order of integration is justified by Fubini's theorem, and the last inequality follows by III, Theorem 5.4 (a). Similarly, 


$$
\begin{aligned}
\int_{x+2 A}^{\infty} G_{N}(x, y) d \mu(y) & =\int_{x+2 A}^{\infty} d_{\mu}(y) \int_{2 A}^{\infty} G_{N}(u) D(x, y, u) d_{\mu}(u) \\
& \leqq \frac{1}{4 A^{2}} \int_{2 A}^{\infty} u^{2} G_{N}(u) d_{\mu}(u) \leqq \frac{1}{4}
\end{aligned}
$$

Applying these inequalities to (2), we have

$$
\int_{x-2 A}^{x+2 A} G_{N}(x, y) d \mu(y) \geqq \frac{1}{2},
$$

or

$$
\begin{aligned}
\frac{1}{2} & \leqq \frac{1}{2^{\gamma-1 / 2} \Gamma(\gamma+1 / 2)} \int_{x-2 A}^{x+2 A} G_{N}(x, y) y^{2 \gamma} d y \\
& \leqq \frac{(x+2 A)^{2 \gamma}}{2^{\gamma-1 / 2} \Gamma(\gamma+1 / 2)} 4 A \operatorname{lic}_{x-2 A \leqq y \leqq x+2 A} G_{N}(x, y),
\end{aligned}
$$

so that

$$
\operatorname{liv.b.}_{x-2 A \leqq y \leqq x+2 A} G_{N}(x, y) \geqq \frac{\Gamma(\gamma+1 / 2)}{2^{1 / 2-\gamma}} \frac{1}{A(x+2 A)^{2 \gamma}} .
$$

Suppose now that $\left|x-\xi_{N}(x)\right|>11 A$.

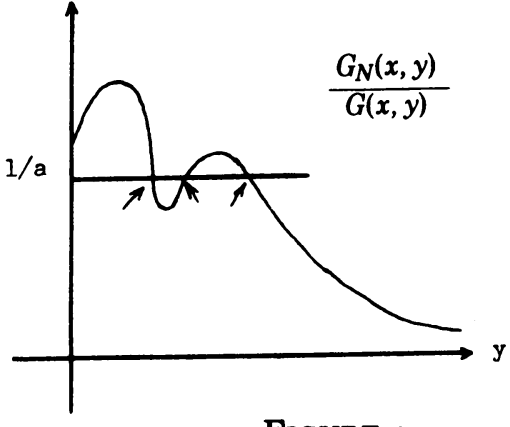

Figure a

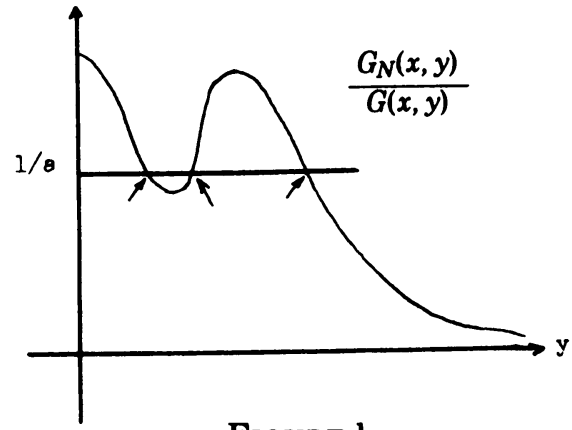

Figure b

Then $G_{N}(x, y) \uparrow$ within the interval $(x-2 A, x+11 A)$ if $x<\xi_{N}(x)$, as in Figure a, and $G_{N}(x, y) \downarrow$ within the interval $(x-11 A, x+2 A)$ if $x>\xi_{N}(x)$, as in Figure b. It therefore follows that (4) holds for $y$ in $(x+2 A, x+11 A)$ and $(x-11 A, x-2 A)$. Hence

$$
\begin{aligned}
\int_{0}^{\infty} G_{N}(x, y) d \mu(y) & \\
\geqq & \frac{1}{2^{\gamma-1,2} \Gamma(\gamma+1 / 2)} \int_{x+2 A}^{x+11 A} G_{N}(x, y) y^{2 \gamma} d y \\
\geqq & \frac{1}{2^{\gamma-1 / 2} \Gamma(\gamma+1 / 2)}\left[\frac{\Gamma(\gamma+1 / 2)}{2^{1 / 2-\gamma}} \frac{1}{A(x+2 A)^{2 \gamma}}\right](x+2 A)^{2 \gamma} 9 A=\frac{9}{8},
\end{aligned}
$$


a contradiction. Similarly,

$$
\begin{aligned}
\int_{0}^{\infty} G_{N}(x, y) d \mu(y) \\
\quad \geqq \frac{1}{2^{\gamma-1 / 2} \Gamma(\gamma+1 / 2)} \int_{x-11 A}^{x-2 A} G_{N}(x, y) y^{2 \gamma} d y \\
\geqq \frac{1}{2^{\gamma-1,2} \Gamma(\gamma+1 / 2)}\left[\frac{\Gamma(\gamma+1 / 2)}{2^{1 / 2-\gamma}} \frac{1}{A(x+2 A)^{2 \gamma}}\right](x-11 A)^{2 \gamma} 9 A \\
\quad=\frac{9}{8}\left(\frac{x-11 A}{x+2 A}\right)^{2 \gamma},
\end{aligned}
$$

and

$$
\liminf _{N \rightarrow \infty} \int_{0}^{\infty} G_{N}(x, y) d \mu(y) \geqq \frac{9}{8},
$$

These contradictions imply that our assumption must be false, so that for $N$ sufficiently large, $\left|x-\xi_{N}(x)\right| \leqq 11 A$, and the theorem holds.

Theorem 3.6. For $N$ large and all $x$ and $y, G_{N}(x, y) \leqq A(x) /\left(s_{N}\right)^{1 / 2}$, where $s_{N}=\sum_{k=N+1}^{\infty}\left(1 / a_{k}^{2}\right)$.

Proof. We have

$$
\begin{aligned}
G_{N}(x, y)= & \int_{0}^{\infty} \frac{\mathscr{f}(x t) \mathscr{f}(y t)}{E_{N}(t)} d \mu(t) \\
= & \frac{1}{2^{\gamma-1 / 2} \Gamma(\gamma+1 / 2)} \int_{0}^{1} \frac{\mathscr{f}(x t) \mathscr{f}(y t)}{E_{N}(t)} t^{2 \gamma} d t \\
& +2^{\gamma-1 / 2} \Gamma(\gamma+1 / 2)(x y)^{1 / 2-\gamma} \int_{1}^{\infty} \frac{J_{\gamma-1 / 2}(x t) J_{\gamma-1 / 2}(y t)}{E_{N}(t)} t d t .
\end{aligned}
$$

Now

$$
E_{N}(t)=\prod_{k=N+1}^{\infty}\left(1+\frac{t^{2}}{a_{k}^{2}}\right)=1+\left(\sum_{k=N+1}^{\infty} \frac{1}{a_{k}^{2}}\right) t^{2}+\left(\sum_{N+1=j<k}^{\infty} \frac{1}{a_{j}^{2} a_{k}^{2}}\right) t^{4}+\cdots,
$$

where the coefficients of powers of $t$ are all positive. Hence $E_{N}(t) \geqq 1+s_{N} t^{2}$. Further, for $0 \leqq u<\infty,|\mathcal{f}(u)| \leqq 1$, and the asymptotic form for $J_{\gamma}(u)$, see $\left[17\right.$, p. 194], gives, for $u>0,\left|J_{\gamma-1 / 2}(u)\right| \leqq c /(u)^{1 / 2}$, where $c$ is a constant. Using these inequalities in the integrals defining $G_{N}(x, y)$, we find that

$$
\begin{aligned}
G_{N}(x, y) & \leqq k_{1} \int_{0}^{1} \frac{d t}{1+s_{N} t^{2}}+k_{2}(x y)^{-\gamma} \int_{1}^{\infty} \frac{d t}{1+s_{N} t^{2}} \\
& \leqq\left[k_{1}+k_{2}(x y)^{-\gamma}\right] \int_{0}^{\infty} \frac{d t}{1+s_{N} t^{2}} \leqq \frac{K(x, y)}{\left(s_{N}\right)^{1,2}} .
\end{aligned}
$$


For $N$ large, if $\xi_{N}(x)$ is the maximum point of $G_{N}(x, y)$, it follows that

$$
G_{N}(x, y) \leqq G_{N}\left(x, \xi_{N}(x)\right) \leqq \frac{K\left(x, \xi_{N}(x)\right)}{\left(s_{N}\right)^{1 / 2}} \leqq \frac{A(x)}{\left(s_{N}\right)^{1 / 2}},
$$

the last inequality following by Theorem 3.5.

4. Application of the differential operator.

Lemma 4.1. For fixed $y$,

$$
\prod_{k=1}^{N}\left(1-\frac{\Delta_{x}}{a_{k}^{2}}\right) G(x, y)=G_{N}(x, y) .
$$

Proof. We have

$$
G(x, y)=\int_{0}^{\infty} \frac{\mathscr{f}(x t) \mathscr{f}(y t)}{E(t)} d \mu(t)
$$

and

$$
G_{N}(x, y)=\int_{0}^{\omega} \frac{\mathscr{J}(x t) \mathscr{f}(y t)}{E_{N}(t)} d_{\mu}(t) .
$$

Applying the operator $\left[\coprod_{k=1}^{N}\left(1-\Delta_{x} / a_{k}^{2}\right)\right.$ to $G(x, y)$ and noting that, by I, $\$ 2,(4)$,

$$
\prod_{k=1}^{N}\left(1-\frac{\Delta_{x}}{a_{k}^{2}}\right) \mathscr{f}(x t)=\prod_{k=1}^{N}\left(1+\frac{t^{2}}{a_{k}^{2}}\right) \mathscr{f}(x t),
$$

we find that

$$
\begin{aligned}
\prod_{k=1}^{N}\left(1-\frac{\Delta_{x}}{a_{k}^{2}}\right) G(x, y) & =\prod_{k=1}^{N}\left(1-\frac{\Delta_{x}}{a_{k}^{2}}\right) \int_{0}^{\infty} \frac{\mathscr{f}(x t) \mathscr{f}(y t)}{E(t)} d \mu(t) \\
& =\int_{0}^{\infty} \frac{\left[\prod_{k=1}^{N}\left(1-\frac{\Delta_{x}}{a_{k}^{2}}\right) \mathscr{f}(x t) \mathscr{f}(y t)\right]}{E(t)} d \mu(t) \\
& =\int_{0}^{\infty} \frac{\prod_{k=1}^{N}\left(1+\frac{t^{2}}{a_{k}^{2}}\right) \mathscr{f}(x t) \mathscr{f}(y t)}{E(t)} d_{\mu}(t) \\
& =\int_{0}^{\infty} \frac{\mathscr{f}(x t) \mathscr{f}(y t)}{E_{N}(t)} d_{\mu}(t)=G_{N}(x, y),
\end{aligned}
$$

where Theorem 2.12 of II justifies moving the operator under the integral sign. 
Theorem 4.2. Let $\phi(x)$ be integrable in every finite interval, and let

$$
f(x)=\int_{0}^{\infty} G(x, y) \phi(y) d \mu(y),
$$

where the integral converges. Then

$$
\prod_{k=1}^{N}\left(1-\frac{\Delta_{x}}{a_{k}^{2}}\right) f(x)=\int_{0}^{\infty} G_{N}(x, y) \phi(y) d \mu(y) .
$$

Proof. By Lemma 4.1,

$$
\prod_{k=1}^{N}\left(1-\frac{\Delta_{x}}{a_{k}^{2}}\right) G(x, y)=G_{N}(x, y),
$$

and hence, by Theorem 2.12 of II, it is sufficient to show that

$$
\int_{0}^{\infty} G_{N}(x, y) \phi(y) d \mu(y)
$$

converges uniformly for $x$ in a finite interval. Now, $G_{N}(x, y) / G(x, y)$ is positive, has one change in trend for $N$ sufficiently large, and $\rightarrow 0$ as $y \rightarrow \infty$. Hence for $y$ large enough, $\left(G_{N}(x, y) / G(x, y)\right) \downarrow$. By the second mean value theorem, we have

$$
\begin{aligned}
\int_{T_{1}}^{T_{2}} G_{N}(x, y) \phi(y) d \mu(y)= & \int_{T_{1}}^{T_{2}} \frac{G_{N}(x, y)}{G(x, y)} G(x, y) \phi(y) d \mu(y) \\
= & \frac{G_{N}\left(x, T_{1}\right)}{G\left(x, T_{1}\right)} \int_{T_{1}}^{w} G(x, y) \phi(y) d \mu(y) \\
& +\frac{G_{N}\left(x, T_{2}\right)}{G\left(x, T_{2}\right)} \int_{w}^{T_{2}} G(x, y) \phi(y) d \mu(y),
\end{aligned}
$$

where $T_{1} \leqq w \leqq T_{2}$. It then follows by Theorem 2.4 and by IV, Theorem 4.1, that

$$
\lim _{T_{1}, T_{2} \rightarrow \infty} \int_{T_{1}}^{T_{2}} G_{N}(x, y) \phi(y) d \mu(y)=0,
$$

uniformly for $x$ in any finite interval, and hence the theorem is proved.

5. Inversion.

Theorem 5.1. Let $\phi(t)$ be a function integrable on every finite interval, and let

$$
f(x)=\int_{0}^{\infty} G_{N}(x, t) \phi(t) d \mu(t), \quad 0<x<\infty,
$$

converge. If 


$$
\lim _{h \rightarrow 0} \frac{1}{h} \int_{x}^{x+h}[\phi(t)-\phi(x)] d \mu(t)=0
$$

then

$$
\lim _{N \rightarrow \infty} \prod_{k=1}^{N}\left(1-\frac{\Delta_{x}}{a_{k}^{2}}\right) f(x)=\phi(x) .
$$

\section{Proof. Let}

$$
I_{N}=\int_{0}^{\infty} G_{N}(x, y)[\phi(y)-\phi(x)] d \mu(y)
$$

The proof will be complete if we can show that $\lim \sup _{N \rightarrow \infty}\left|I_{N}\right|=0$. Let

$$
\psi(y)=\int_{x}^{y}[\phi(t)-\phi(x)] d \mu(t)
$$

so that

$$
\psi^{\prime}(y) d y=[\phi(y)-\phi(x)] d_{\mu}(y) .
$$

From the hypothesis, it follows that for a given $\epsilon>0$, there is a $\delta>0$ such that

$$
|\psi(y)| \leqq\left|\int_{x}^{y}[\phi(t)-\phi(x)] d_{\mu}(t)\right| \leqq \epsilon|y-x|,
$$

for $|y-x|<\delta$. With $x$ and $\delta$ fixed, $\delta<x / 2$, choose $N$ so large that $G_{N}(x, y)$ has its one change of trend at $\xi_{N}(x)$ within the interval $(x-\delta / 2, x+\delta / 2)$. Now let

$$
\begin{aligned}
I_{N} & =\left(\int_{x}^{x-\delta}+\int_{x-\delta}^{x+\delta}+\int_{x+\delta}^{\infty}\right) G_{N}(x, y)[\phi(y)-\phi(x)] d \mu(y) \\
& =I_{1}+I_{2}+I_{3} .
\end{aligned}
$$

Since $G_{N}(x, y) \uparrow$ in $(0, x-\delta)$, we have, by the mean value theorem,

$$
\begin{aligned}
I_{1} & =\int_{0}^{x-\delta} G_{N}(x, y)[\phi(y)-\phi(x)] d_{\mu}(y) \\
& =G_{N}(x, x-\delta) \int_{y}^{x-\delta}[\phi(y)-\phi(x)] d \mu(y),
\end{aligned}
$$

where $0 \leqq y \leqq x-\delta$. Hence, by Theorem 2.7 ,

$$
\lim _{N \rightarrow \infty} I_{1}=0 \text {. }
$$

Also, using the mean value theorem again, we find that 


$$
\begin{aligned}
I_{3} & =\int_{x+\delta}^{\infty} G_{N}(x, y)[\phi(y)-\phi(x)] d \mu(y) \\
& =\int_{x+\delta}^{\infty} \frac{G_{N}(x, y)}{G(x, y)} G(x, y) \phi(y) d \mu(y)-\phi(x) \int_{x+\delta}^{\infty} G_{N}(x, y) d_{\mu}(y) \\
& =\frac{G_{N}(x, x+\delta)}{G(x, x+\delta)} \int_{x+\delta}^{\xi} G(x, y) \phi(y) d \mu(y)-\phi(x) \int_{x+\delta}^{\infty} G_{N}(x, y) d_{\mu}(y),
\end{aligned}
$$

where $x+\delta \leqq \xi<\infty$. By Theorem 2.7, and by III, Theorem 5.7, we have (7)

$$
\lim _{N \rightarrow \infty} I_{3}=0 \text {. }
$$

Further,

$$
\begin{aligned}
I_{2}= & \int_{x-\delta}^{x+\delta} G_{N}(x, y)[\phi(y)-\phi(x)] d \mu(y) \\
= & \int_{x-\delta}^{x+\delta} G_{N}(x, y) \psi^{\prime}(y) d y \\
= & G_{N}(x, x+\delta) \psi(x+\delta)-G_{N}(x, x-\delta) \psi(x-\delta) \\
& -\int_{x-\delta}^{x+\delta} \psi(y) \frac{d}{d y} G_{N}(x, y) d y .
\end{aligned}
$$

Hence, by Theorem 2.7,

$$
\left|I_{2}\right| \leqq o(1)+\int_{x-\delta}^{x+\delta}|\psi(y)|\left|\frac{d}{d y} G_{N}(x, y)\right| d y
$$

as $N \rightarrow \infty$. By (4),

$$
\begin{aligned}
& \left|I_{2}\right| \leqq o(1)+\epsilon \int_{x-\delta}^{x+\delta}|y-x|\left|\frac{d}{d y} G_{N}(x, y)\right| d y \\
& \leqq 0(1)+\epsilon \int_{x-\delta}^{x+\delta}\left(\left|y-\xi_{N}(x)\right|+\left|\xi_{N}(x)-x\right|\right)\left|\frac{d}{d y} G_{N}(x, y)\right| d y \\
& \text { (8) } \leqq o(1)+\epsilon\left\{\int_{x-\delta}^{x+\delta}\left(\xi_{N}(x)-y\right) \frac{d}{d y} G_{N}(x, y) d y\right. \\
& +\left|\xi_{N}(x)-x\right|\left[\int_{x-\delta}^{\xi_{N}^{(x)}} \frac{d}{d y} G_{N}(x, y) d y\right. \\
& \left.\left.-\int_{\xi_{N}(x)}^{x+\delta} \frac{d}{d y} G_{N}(x, y) d y\right]\right\} \text {. }
\end{aligned}
$$

Now, on integrating by parts, we have, as $N \rightarrow \infty$, 


$$
\begin{aligned}
\int_{x-\delta}^{x+\delta}( & \left.\xi_{N}(x)-y\right) \frac{d}{d y} G_{N}(x, y) d y \\
& =o(1)+\int_{x-\delta}^{x+\delta} G_{N}(x, y) d y \\
& =o(1)+\frac{\Gamma(\gamma+1 / 2)}{2^{1 / 2-\gamma}} \int_{x-\delta}^{x+\delta} \frac{1}{y^{2 \gamma}} G_{N}(x, y) d \mu(y) \\
& \leqq o(1)+\frac{\Gamma(\gamma+1 / 2)}{2^{1 / 2-\gamma}(x-\delta)^{2 \gamma}} \int_{x-\delta}^{x+\delta} G_{N}(x, y) d \mu(y) \\
& \leqq o(1)+\frac{\Gamma(\gamma+1 / 2)}{2^{1 / 2-3 \gamma} x^{2 \gamma}} \\
& =o(1)+K(x),
\end{aligned}
$$

where the last inequality follows since $\delta$ was chosen $<x / 2$. Also,

$$
\begin{gathered}
\int_{x-\delta}^{\xi_{N}} \frac{d}{d y} G_{N}(x, y) d y-\int_{\xi_{N}}^{x+\delta} \frac{d}{d y} G_{N}(x, y) d y \\
=2 G_{N}\left(x, \xi_{N}(x)\right)+o(1), \quad N \rightarrow \infty .
\end{gathered}
$$

Applying (9) and (10) to (8), we find that

$\left|I_{2}\right| \leqq o(1)+\epsilon\left\{o(1)+K(x)+\left|\xi_{N}(x)-x\right|\left[2 G_{N}\left(x, \xi_{N}(x)\right)+o(1)\right]\right\}, \quad N \rightarrow \infty$.

By Theorems 3.5 and 3.6,

$$
\underset{N=0,1, \cdots}{\operatorname{l.u.b}}\left|\xi_{N}(x)-x\right| G_{N}\left(x, \xi_{N}(x)\right)=B(x)
$$

is finite. If we select, for arbitrary $\epsilon^{\prime}>0$,

$$
\epsilon \leqq \frac{\epsilon^{\prime}}{K(x)+2 B(x)}
$$

it follows that

$$
\limsup _{N \rightarrow \infty}\left|I_{2}\right| \leqq \epsilon^{\prime}
$$

and hence, combining (6), (7) and (11), we have limsup $\sup _{N \rightarrow \infty}\left|I_{N}\right| \leqq \epsilon^{\prime}$, and the theorem follows.

\section{Chapter VI. Representation}

1. Introduction. In this chapter, we develop a representation theory corresponding to the inversion theory. We find that we must consider the Stieltjes integral $\int_{0}{ }^{\infty} G(x, y) d \psi(y)$ rather than $\int_{0}{ }^{\infty} G(x, y) \phi(y) d_{\mu}(y)$. Our major preceding results, however, may be established equally well for the 
Stieltjes integral. In particular, if

$$
f(x)=\int_{0}^{\infty} G(x, y) d \psi(y)
$$

converges conditionally for any value of $x, 0 \leqq x<\infty$, then it converges for all such $x$ and uniformly for $x$ in any finite interval. Further, by the Stieltjes form of $\mathrm{V}$, Theorem $4.2, f(x)$ is infinitely differentiable for $0 \leqq x$ $<\infty$ and

(2) $\prod_{k=1}^{N}\left(1-\frac{\Delta_{x}}{a_{k}^{2}}\right) f(x)=\int_{0}^{\infty} G_{N}(x, y) d \psi(y), \quad 0 \leqq x<\infty, N=0,1, \cdots$

Note that if $\psi(y) \uparrow$, since $G_{N}(x, y)>0$,

$$
\prod_{k=1}^{N}\left(1-\frac{\Delta_{x}}{a_{k}^{2}}\right) f(x) \geqq 0, \quad 0 \leqq x<\infty, N=0,1, \cdots .
$$

It is our aim to show that necessary and sufficient conditions for a function $f$ to have the representation (1) with $\psi(y) \uparrow$ are that $f(x)$ be infinitely differentiable for $0 \leqq x<\infty$, that (3) hold and that $f(x)=o\left(\mathcal{I}\left(a_{1} x\right)\right), x \rightarrow \infty$.

2. Behavior at infinity. If the *-convolution transform

$$
f(x)=\int_{-\infty}^{\infty} G(x-t) d \alpha(t)
$$

converges for some value of $x$, where $G$ is a variation diminishing *-kernel, it has been shown, [10, p. 147] that $f(x)=o\left(e^{\alpha x}\right), x \rightarrow \infty$. We derive an analogous result for the \#-convolution.

TheOREM 2.1. Let $f(x)=\int_{0}^{\infty} G(x, t) d \psi(t), 0 \leqq x<\infty$, converge. Then $f(x)$ $=o\left(\mathscr{P}\left(a_{1} x\right)\right), x \rightarrow \infty$.

Proof. Let $s(y)=\int_{y}^{\infty} G(0, u) d \psi(u)$ and $t(y)=G(x, y) / G(0, y)$. By hypothesis, it follows that, for a given $\epsilon>0$, there exists a $y_{0}$ such that $|s(y)|\left\langle\epsilon, y \geqq y_{0}\right.$. Further, by III, Theorem 4.4, $t(y) \uparrow$, and by IV, Theorem 3.2, $\lim _{y \rightarrow \infty} t(y)=\mathscr{P}\left(a_{1} x\right)$. Now let $f(x)=f_{1}(x)+f_{2}(x)$, where $f_{1}(x)$ $=\int_{0}^{y_{0}} G(x, y) d \psi(y)$ and $f_{2}(x)=\int_{y_{0}}^{\infty} G(x, y) d \psi(y)$. Since $(G(x, y) / G(0, y)) \uparrow$, on applying the mean value theorem, we have

$$
\begin{aligned}
f_{1}(x) & =\int_{0}^{y_{0}} \frac{G(x, y)}{G(0, y)} G(0, y) d \psi(y) \\
& =\frac{G\left(x, y_{0}\right)}{G\left(0, y_{0}\right)} \int_{\eta}^{y_{0}} G(0, y) d \psi(y),
\end{aligned}
$$

where $0 \leqq \eta \leqq y_{0}$. Then IV, Theorem 3.2 implies that, as $x \rightarrow \infty, f_{1}(x)=o(1)$. Further, 


$$
\begin{aligned}
f_{2}(x) & =\int_{y_{0}}^{\infty} \frac{G(x, y)}{G(0, y)} G(0, y) d \psi(y) \\
& =-\int_{y_{0}}^{\infty} t(y) d s(y) \\
& =t\left(y_{0}\right) s\left(y_{0}\right)+\int_{y_{0}}^{\infty} s(y) d t(y) \\
& \leqq \epsilon t\left(y_{0}\right)+\epsilon \int_{y_{0}}^{\infty} d t(y) \leqq \epsilon\left(\mathcal{P}\left(a_{1} x\right)\right),
\end{aligned}
$$

so that $f(x)=o\left(\mathcal{P}\left(a_{1} x\right)\right), x \rightarrow \infty$.

3. A basic representation theorem. In this section we develop a fundamental representation theorem which will be needed to derive our major result.

LEMMA 3.1. Let

$$
H_{1}(x, y)=\int_{0}^{\infty} \frac{\mathscr{f}(x t) \mathscr{f}(y t)}{1+\frac{t^{2}}{a_{k}^{2}}} d_{\mu}(t), \quad 0 \leqq x, y<\infty .
$$

Then

$$
\begin{aligned}
H_{1}(x, y) & =a^{2} 2^{\gamma-1 / 2} \Gamma(\gamma+1 / 2) \mathscr{Y}(a x) \mathscr{P}(a y) \int_{y}^{\infty} \frac{d t}{t^{2 \gamma}[\mathscr{Y}(a t)]^{2}} \text { if } x \leqq y, \\
& =a^{2} 2^{\gamma-1 / 2} \Gamma(\gamma+1 / 2) \mathscr{Y}(a x) \mathscr{P}(a y) \int_{x}^{\infty} \frac{d t}{t^{2 \gamma}[\mathscr{I}(a t)]^{2}} \text { if } x \geqq y .
\end{aligned}
$$

Proof. We have by III, Lemma 3.3,

$$
\mathscr{K}(a x) \frac{d}{d x} \mathscr{P}(a x)-\mathscr{P}(a x) \frac{d}{d x} \mathscr{K}(a x)=\frac{2^{\gamma-1 / 2} \Gamma(\gamma+1 / 2)}{x^{2 \gamma} a^{2 \gamma-1}} .
$$

Dividing both sides by $[\mathcal{H}(a x)]^{2}$ and integrating from $y$ to $\infty$, we have, since $\lim _{x \rightarrow \infty}(\mathscr{K}(a x) / \mathscr{P}(a x))=0$,

$$
\frac{\mathscr{K}(a y)}{\mathscr{I}(a y)}=\frac{2^{\gamma-1 / 2} \Gamma(\gamma+1 / 2)}{a^{2 \gamma-1}} \int_{y}^{\infty} \frac{d x}{x^{2 \gamma}[\mathscr{I}(a x)]^{2}} .
$$

But, by III, Lemma 3.4,

$$
\begin{aligned}
H_{1}(x, y) & =a^{2 \gamma+1} \mathscr{K}(a y) \mathscr{I}(a x), & & x \leqq y, \\
& =a^{2 \gamma+1} \mathscr{K}(a x) \mathscr{I}(a y), & & x \geqq y .
\end{aligned}
$$

Substituting (2) in (3), we have the desired result. 
LEMMA 3.2. Let $\Phi(t)$ be a function integrable in every finite interval and non-negative for $0 \leqq x<\infty$. Then

$$
\int_{0}^{\infty} H(x, t) \Phi(t) d_{\mu}(t)=\int_{x}^{\infty} \frac{a^{2} \mathscr{P}(a x)}{u^{2 \gamma}[\mathscr{I}(a u)]^{2}} d u \int_{0}^{u} t^{2 \gamma} \mathscr{Y}(a t) \Phi(t) d t,
$$

whenever either side is finite.

Proof. Since, clearly, $H(x, y)=H(y, x)$, we have, on applying Lemma 3.1,

$$
\begin{aligned}
\int_{0}^{\infty} H(x, t) \Phi(t) d \mu(t) \\
=\int_{0}^{x} H(t, x) \Phi(t) \frac{t^{2 \gamma} d t}{2^{\gamma-1 / 2} \Gamma(\gamma+1 / 2)} \\
\quad+\int_{x}^{\infty} H_{1}(x, t) \Phi(t) \frac{t^{2 \gamma} d t}{2^{\gamma-1 / 2} \Gamma(\gamma+1 / 2)} \\
=\int_{0}^{x} t^{2 \gamma} \Phi(t) a^{2} \mathscr{P}(a x) \mathscr{P}(a t) d t \int_{x}^{\infty} \frac{d u}{u^{2 \gamma}[\mathscr{P}(a u)]^{2}} \\
\quad+\int_{x}^{\infty} t^{2 \gamma} \Phi(t) a^{2} \mathscr{P}(a x) \mathscr{P}(a t) d t \int_{t}^{\infty} \frac{d u}{u^{2 \gamma}[\mathscr{P}(a u)]^{2}} .
\end{aligned}
$$

By Fubini's theorem, the order of integration may be inverted to give the desired result.

Lemma 3.3. Let $f(x) \in C^{2}, 0 \leqq x<\infty ; f(x)=o(\mathscr{P}(a x)), a>0, x \rightarrow \infty ; f^{\prime \prime}(x)$ $\geqq O(\mathscr{P}(a x)), x \rightarrow \infty$. Then $f^{\prime}(x)=o(\mathscr{P}(a x))$.

Proof. On integrating by parts, we may verify that for any real number $\theta$,

$$
f^{\prime}(x)=\frac{f(x+\theta)-f(x)}{\theta}+\frac{1}{\theta} \int_{x}^{x+\theta}(t-x-\theta) f^{\prime \prime}(t) d t .
$$

Since $f^{\prime \prime}(x) \geqq O(\mathscr{I}(a x)), x \rightarrow \infty$, there exists a non-negative number $A$ such that

$$
f^{\prime \prime}(x) \geqq-A \mathscr{I}(a x), \quad 0 \leqq x<\infty,
$$

and hence

$$
\begin{aligned}
f^{\prime}(x) & \leqq o(\mathscr{F}(a x))+A \theta \mathscr{Y}(a(x+\theta)), \quad x \rightarrow \infty, \theta>0, \\
& \geqq o(\mathscr{F}(a x))+A \theta \mathscr{Y}(a x), \quad x \rightarrow \infty, \theta<0 .
\end{aligned}
$$

Since $\theta$ may be chosen arbitrarily small, it follows that

$$
f^{\prime}(x)=o(\mathscr{F}(a x)), \quad x \rightarrow \infty,
$$

as was to be shown. 
LemMa 3.4. Let

$$
Q(D)=q_{n}(x) D^{n}+q_{n-1}(x) D^{n-1}+\cdots+q_{0}(x), \quad 0<x<\infty,
$$

be a linear differential operator of degree $n$ with coefficients such that the $q_{i}^{(j)}(x), j=0,1, \cdots, i, i=0,1, \cdots, n$ are all bounded for $X_{0} \leqq<\infty$ for any $X_{0}>0$. If $f(x) \in C^{n}, 0 \leqq x<\infty ; f(x)=o(\mathscr{F}(a x)), a>0, x \rightarrow \infty ; Q(D) f(x)$ $\geqq O(\mathscr{F}(a x)), \quad x \rightarrow \infty$, then $f^{(k)}(x)=o(\mathscr{Y}(a x)), \quad x \rightarrow \infty, k=1,2, \cdots, n-1$.

Proof. It is sufficient to prove the theorem for $k=1$. For, suppose the theorem holds for $k=1$, so that with the given hypotheses, we have $f^{\prime}(x)$ $=o(\mathscr{Y}(a x)), x \rightarrow \infty$. Let $Q^{*}(D)=q_{n}(x) D^{n-1}+\cdots+q_{1}(x)$. Then

$$
\begin{aligned}
Q^{*}(D) D(f(x)) & =Q^{*}(D) f^{\prime}(x) \\
& =Q(D) f(x)-q_{0}(x) f(x) .
\end{aligned}
$$

Since $q_{0}(x)$ is bounded, this implies that $Q^{*}(D) f^{\prime}(x) \geqq O(\mathscr{I}(a x)), x \rightarrow \infty$. But, applying the theorem, which we assume holds for $k=1$, to $f^{\prime}(x)$ and the operator $Q^{*}(D)$, it follows that $f^{\prime \prime}(x)=o(\mathscr{I}(a x)), x \rightarrow \infty$. Proceeding in this way, we may clearly conclude that $f^{(k)}(x)=o(\mathscr{P}(a x)), x \rightarrow \infty$, $k=2, \cdots, n-1$. It thus remains for us to establish the theorem only for $k=1$. To this end, set

$$
F(x)=\int_{x_{0}}^{x}(x-t)^{n-1} Q(D) f(t) d t, \quad x_{0}>0 .
$$

On integrating by parts, we find that

$$
\begin{aligned}
F(x)= & P_{n-1}(x)+(n-1) ! q_{n}(x) f(x) \\
& +\sum_{k=0}^{n}(-1)^{k} \int_{x_{0}}^{x}\left[(x-t)^{n-1} q_{k}(t)\right]^{(k)} f(t) d t,
\end{aligned}
$$

where $P_{n-1}(x)$ is a polynomial of degree $n-1$. Since the $q_{i}^{(j)}, j=0, \cdots, i$, $i=0, \cdots, n$ are bounded for $x_{0} \leqq x<\infty$ and since $f(x)=o(\mathscr{Y}(a x)), x \rightarrow \infty$, it follows from (2) that

$$
F(x)=o(\mathscr{P}(a x)), \quad x \rightarrow \infty .
$$

Further, differentiating (1) twice, we have

$$
F^{\prime \prime}(x)=(n-1)(n-2) \int_{x_{0}}^{x}(x-t)^{n-3} Q(D) f(t) d t,
$$

and since $Q(D) f(x) \geqq O(\mathscr{Y}(a x))$, we find that

$$
F^{\prime \prime}(x) \geqq O(\mathscr{P}(a x)), \quad x \rightarrow \infty .
$$

$F(x)$ thus satisfies the conditions of Lemma 3.2 and hence 


$$
F^{\prime}(x)=o(\mathscr{F}(a x)), \quad x \rightarrow \infty .
$$

But, on differentiating (2), we find that

$$
F^{\prime}(x)=(n-1) ! q_{n}(x) f^{\prime}(x)+o(\mathcal{Y}(a x)), \quad x \rightarrow \infty,
$$

and since $q_{n}(x)$ is bounded, it follows that $f^{\prime}(x)=o(\mathscr{I}(a x)), x \rightarrow \infty$, and the lemma is proved.

Lemma 3.5. Let (a) $f(x) \in C^{2}, 0 \leqq x<\infty$, (b) $f(x)=o\left(\mathscr{I}\left(a_{1} x\right)\right), x \rightarrow \infty$. Then

$$
f(x)=\int_{x}^{\infty} \frac{a_{1}^{2} \mathscr{F}\left(a_{1} x\right)}{u^{2 \gamma}\left[\mathscr{I}\left(a_{1} u\right)\right]^{2}} d u \int_{0}^{u} t^{2 \gamma} \mathscr{F}\left(a_{1} t\right)\left[\left(1-\frac{\Delta_{t}}{a_{1}^{2}}\right)\right] f(t) d t .
$$

Proof. Note that the assumption that $\left[1-\Delta_{x} / a_{1}^{2}\right] f(0)$ is defined implies that $f^{\prime}(0)=0$. By III, Lemma 3.2, we have

(1) $\left[1-\frac{\Delta_{x}}{a_{1}^{2}}\right] f(x)=-\frac{1}{a_{1}^{2} x^{2 \gamma} \mathscr{Y}\left(a_{1} x\right)} \frac{d}{d x}\left[x^{2 \gamma}\left[\mathscr{F}\left(a_{1} x\right)\right]^{2} \frac{d}{d x}\left(\frac{f(x)}{\mathscr{I}\left(a_{1} x\right)}\right)\right]$.

Multiplying each side of (1) by $a_{1}^{2} x^{2 \gamma} \mathscr{I}\left(a_{1} x\right)$ and integrating with respect to $x$ from 0 to $u$, then dividing both sides by $u^{2 \gamma}\left[\mathscr{I}\left(a_{1} u\right)\right]^{2}$ and integrating with respect to $u$ from 0 to $x$, we find that

(2) $f(x)=\mathscr{I}\left(a_{1} x\right)\left\{f(0)-\int_{0}^{x} \frac{a_{1}^{2} d u}{u^{2 \gamma}\left[\mathscr{I}\left(a_{1} u\right)\right]^{2}} \int_{0}^{u} t^{2 \gamma} \mathscr{I}\left(a_{1} t\right)\left[1-\frac{\Delta_{t}}{a_{1}^{2}}\right] f(t) d t\right\}$.

As a result of hypothesis (b), it is clear that

$$
f(0)=\int_{0}^{\infty} \frac{a_{1}^{2} d u}{u^{2 \gamma}\left[\mathscr{I}\left(a_{1} u\right)\right]^{2}} \int_{0}^{u} t^{2 \gamma} \mathscr{Y}\left(a_{1} t\right)\left[\left(1-\frac{\Delta_{t}}{a_{1}^{2}}\right) f(t)\right] d t
$$

and hence

(4) $\quad f(x)=\int_{x}^{\infty} \frac{a_{1}^{2} \mathscr{F}\left(a_{1} x\right)}{u^{2 \gamma}\left[\mathscr{I}\left(a_{1} u\right)\right]^{2}} d u \int_{0}^{u} t^{2 \gamma} \mathscr{F}\left(a_{1} t\right)\left[\left(1-\frac{\Delta_{t}}{a_{1}^{2}}\right) f(t)\right] d t$.

ThEOREM 3.6. Let (a) $f(x) \in C^{\infty}, 0 \leqq x<\infty$, (b) $f(x)=o\left(\mathcal{I}\left(a_{1} x\right)\right), x \rightarrow \infty$, (c) $\coprod_{k=1}^{N}\left(1-\Delta_{x} / a_{k}^{2}\right) f(x) \geqq 0,0 \leqq x<\infty, N=1,2, \cdots$ Then

$$
f(x)=\int_{0}^{\infty} G_{N}^{*}(x, y)\left[I_{k=1}^{N}\left(1-\frac{\Delta_{y}}{a_{k}^{2}}\right) f(y)\right] d \mu(y), \quad 0 \leqq x<\infty, N=1,2, \cdots,
$$

where

$$
G_{N}^{*}(x, y)=\int_{0}^{\infty} \frac{\mathscr{f}(x t) \mathscr{f}(y t)}{\prod_{k=1}^{N}\left(1+\frac{t^{2}}{a_{k}^{2}}\right)} d \mu(t), \quad 0 \leqq x, y<\infty, N=1,2, \cdots
$$


Proof. We note that by Lemma 3.4,

$$
f^{(k)}(x)=o\left(\mathscr{Y}\left(a_{1} x\right)\right), \quad x \rightarrow \infty, k=1,2, \cdots .
$$

Now if

$$
f_{N}(x)=\prod_{k=1}^{N}\left(1-\frac{\Delta_{x}}{a_{k}^{2}}\right) f(x), \quad 0 \leqq x<\infty,
$$

then clearly

$$
f_{N}(x)=\left(1-\frac{\Delta_{x}}{a_{N}^{2}}\right) f_{N-1}(x)
$$

Since, as a result of $(1), f_{N-1}(x)$ satisfies the conditions of Lemma 3.5, we have

$$
f_{N-1}(x)=\int_{x}^{\infty} \frac{a_{N}^{2} \mathscr{Y}\left(a_{N} x\right)}{u_{N}^{2}\left[\mathscr{I}\left(a_{N} u_{N}\right)\right]^{2}} d u_{N} \int_{0}^{u_{N}} t_{N}^{2 \gamma} \mathscr{Y}\left(a_{N} t_{N}\right) f_{N}\left(t_{N}\right) d t_{N}
$$

Now writing

$$
f_{N-1}(x)=\left(1-\frac{\Delta_{x}}{a_{N-1}^{2}}\right) f_{N-2}(x)
$$

and repeating the argument, we find that

$$
\begin{aligned}
f_{N-2}(x)= & \int_{x}^{\infty} \frac{a_{N-1}^{2} \mathcal{F}\left(a_{N-1} x\right)}{u_{N-1}^{2}\left[\mathcal{F}\left(a_{N-1} u_{N-1}\right)\right]^{2}} d u_{N-1} \int_{0}^{u} t_{N-1}^{2 \gamma} \mathcal{F}\left(a_{N-1} t_{N-1}\right) f_{N-1}\left(t_{N-1}\right) d t_{N-1} \\
= & \int_{x}^{\infty} \frac{a_{N-1}^{2} \mathcal{F}\left(a_{N-1} x\right)}{u_{N-1}^{2}\left[\mathcal{F}\left(a_{N-1} u_{N-1}\right)\right]^{2}} d u_{N-1} \int_{0}^{u} t_{N-1}^{2 \gamma} \mathcal{Y}\left(a_{N-1} t_{N-1}\right) d t_{N-1} \\
& \cdot \int_{t_{N-1}}^{\infty} \frac{a_{N}^{2} \mathcal{F}\left(a_{N} t_{N-1}\right)}{u_{N}^{2}\left[\mathcal{F}\left(a_{N} u_{N}\right)\right]^{2}} d u_{N} \int_{0}^{u_{N}} t_{N}^{2 \gamma} \mathscr{F}\left(a_{N} t_{N}\right) f_{N}\left(t_{N}\right) d t_{N .}
\end{aligned}
$$

Proceeding in this manner, we finally express $f(x)$ in terms of $2 N$ iterated integrals

$$
\begin{aligned}
& f(x)=\int_{x}^{\infty} \frac{a_{1}^{2} \mathscr{F}\left(a_{1} x\right)}{u_{1}^{2}\left[\mathscr{F}\left(a_{1} u\right)\right]^{2}} d u_{1} \int_{0}^{u_{1}} t_{1}^{2 \gamma} \mathscr{F}\left(a_{1} t_{1}\right) d t_{1} \int_{t_{1}}^{\infty} \frac{a_{2}^{2} \mathscr{Y}\left(a_{2} t_{1}\right)}{u_{2}^{2}\left[\mathscr{F}\left(a_{2} u_{2}\right)\right]^{2}} d u_{2} \\
& \cdot \int_{0}^{u_{2}} t_{2}^{2 \gamma} \mathscr{I}\left(a_{2} t_{2}\right) d t_{2} \ldots \int_{t_{N-1}}^{\infty} \frac{a_{N}^{2} \mathscr{I}\left(a_{N} t_{N-1}\right)}{u_{N}^{2}\left[\mathscr{I}\left(a_{N} u_{N}\right)\right]^{2}} d u_{N} \int_{0}^{u} t^{2 \gamma} \mathscr{I}\left(a_{N} t_{N}\right) \\
& \cdot \prod_{k=1}^{N}\left(1-\frac{\Delta_{t_{N}}}{a_{k}^{2}}\right) f\left(t_{N}\right) d t_{N}
\end{aligned}
$$

Condition (c) now enables us to apply Lemma 3.2 repeatedly with the result that 


$$
f(x)=\int_{0}^{\infty} H_{1}\left(x, t_{1}\right) d \mu\left(t_{1}\right) \int_{0}^{\infty} H_{2}\left(t_{1}, t_{2}\right) d \mu\left(t_{2}\right) \cdots \int_{0}^{\infty} H_{N-1}\left(t_{N-2}, t_{N-1}\right) d \mu\left(t_{N-1}\right)
$$

(5)

$$
\cdot \int_{0}^{\infty} H_{N}\left(t_{N-1}, t_{N}\right)\left[\prod_{k=1}^{N}\left(1-\frac{\Delta_{t_{N}}}{a_{k}^{2}}\right) f\left(t_{N}\right)\right] d_{\mu}\left(t_{N}\right)
$$

where

$$
H_{N}(x, y)=\int_{0}^{\infty} \frac{\mathscr{f}(x t) \mathscr{f}(y t)}{1+\frac{t^{2}}{a_{N}^{2}}} d \mu(t)
$$

By V, Lemma 2.2, and Fubini's theorem, it now follows that

$$
f(x)=\int_{0}^{\infty} G_{N}^{*}(x, t)\left[\prod_{k=1}^{N}\left(1-\frac{\Delta_{t}}{a_{k}^{2}}\right) f(t)\right] d_{\mu}(t),
$$

where $G_{N}^{*}(x, t)$ is defined as above.

Corollary 3.7. Let (a) $f(x) \in C^{2 N}, 0 \leqq x<\infty, N=1,2, \cdots$, (b) $f(x)$ $=o\left(\mathscr{I}\left(a_{1} x\right)\right), \quad x \rightarrow \infty, \quad$ (c) $\coprod_{k=1}^{N_{i}}\left(1-\Delta_{x} / a_{k}^{2}\right) f(x) \geqq 0, \quad 0 \leqq x<\infty, 1=N_{0}$ $<N_{1}<\ldots$. Then

$f(x)=\int_{0}^{\infty} G_{N_{i}}^{*}(x, y) \prod_{k=1}^{N_{i}}\left(1-\frac{\Delta_{y}}{a_{k}^{2}}\right) f(y) d \mu(y), \quad 0 \leqq x<\infty, 1=N_{0}<N_{1}<\cdots$

Proof. With the weaker conditions of the corollary the proof of the theorem may be followed to the point where $f(x)$ is expressed by the iterated integrals (4). Lemma 3.2, in this case however, can be applied only for those values of $N$ in the sequence $N_{0}, N_{1}, \cdots$ and hence $f(x)$ has the representation given above.

4. Representation.

LEMMa 4.1. Let $G_{N}^{*}(x, y)$ be defined as in Theorem 3.6. Then $\lim _{N \rightarrow \infty} G_{N}^{*}(x, y)$ $=G(x, y)$ uniformly for $x$ fixed, $0 \leqq x<\infty$, and for $y$ in any finite interval.

Proof. Consider

$$
\begin{aligned}
\left|G_{N}^{*}(x, y)-G(x, y)\right| & \\
& =\left|\int_{0}^{\infty} f(x t) \mathscr{f}(y t)\left[\frac{1}{\prod_{k=1}^{N}\left(1+\frac{t^{2}}{a_{k}^{2}}\right)}-\frac{1-}{\prod_{k=1}^{\infty}\left(1+\frac{t^{2}}{a_{k}^{2}}\right)}\right] d \mu(t)\right| \\
& \leqq \int_{0}^{\infty}\left|\frac{1}{\prod_{k=1}^{N}\left(1+\frac{t^{2}}{a_{k}^{2}}\right)}-\frac{1}{\prod_{k=1}^{\infty}\left(1+\frac{t^{2}}{a_{k}^{2}}\right)}\right| d_{\mu}(t) .
\end{aligned}
$$


Now

$$
\lim _{N \rightarrow \infty}\left|\frac{1}{\prod_{k=1}^{N}\left(1+\frac{t^{2}}{a_{k}^{2}}\right)}-\frac{1}{\prod_{k=1}^{\infty}\left(1+\frac{t^{2}}{a_{k}^{2}}\right)}\right|=0,
$$

and, for $t \geqq 1$ and all $N \geqq 2 \gamma+1$, we have

$$
\begin{aligned}
\left|\frac{1}{\prod_{k=1}^{N}\left(1+\frac{t^{2}}{a_{k}^{2}}\right)}-\frac{1}{\prod_{k=1}^{\infty}\left(1+\frac{t^{2}}{a_{k}^{2}}\right)}\right| & \leqq \frac{2}{\prod_{k=1}^{N}\left(1+\frac{t^{2}}{a_{k}^{2}}\right)} \\
& \leqq \frac{2}{1+c t^{2 N}} \leqq \frac{2}{1+c t^{4 \gamma+2}},
\end{aligned}
$$

$c<0$, so that Lebesgue's limit theorem may be applied to obtain

$$
\lim _{N \rightarrow \infty} \int_{0}^{\infty}\left|\frac{1}{\prod_{k=1}^{N}\left(1+\frac{t^{2}}{a_{k}^{2}}\right)}-\frac{1}{\prod_{k=1}^{\infty}\left(1+\frac{t^{2}}{a_{k}^{2}}\right)}\right| d_{\mu}(t)=0,
$$

whence the result.

Helly's first and second theorems are proved in [11, Vol. I, pp. 222, 233]. We find it useful to combine and restate them in the following forms:

Theorem 4.2. Let $f(x)$ be a continuous function defined for $0 \leqq x<\infty$ and such that $f(\infty)$ exists. Let $g_{n}(x)$ be a sequence of functions, defined on $0 \leqq x<\infty$, which are uniformly bounded and which are of total variation uniformly bounded by $K$. Then there exists a subsequence $g_{n_{i}}(x)$ of the sequence $g_{n}(x)$ converging to a function $g(x)$ at every point of continuity of $g(x) . g(x)$ is of total variation bounded by $K$ and

$$
\lim _{n_{i} \rightarrow \infty} \int_{0}^{\infty} f(x) d g_{n_{i}}(x)=\int_{0}^{\infty} f(x) d g(x)+c f(\infty) .
$$

We are now in a position to derive our main representation theorem.

Theorem 4.3. Necessary and sufficient conditions that

$$
f(x)=\int_{0}^{\infty} G(x, t) d \psi(t)
$$

with $\psi(t) \uparrow$ are that

(a) $f(x) \in C^{\infty}, 0 \leqq x<\infty$,

(b) $\prod_{k=1}^{N_{i}}\left(1-\Delta_{x} / a_{k}^{2}\right) f(x) \geqq 0,0 \leqq x<\infty, 1 \leqq N_{0}<N_{1}<\cdots$,

(c) $f(x)=o\left(\mathscr{I}\left(a_{1} x\right)\right), x \rightarrow \infty$.

Proof. The necessity of conditions (a) and (b) have already been dealt with in the introduction to the chapter, and of condition (c) in Theorem 
2.1. It thus remains to establish the sufficiency of the conditions. By Corollary 3.7 , we have

$$
f(x)=\int_{0}^{\infty} G_{N_{i}}^{*}(x, t)\left[\prod_{k=1}^{N_{i}}\left(1-\frac{\Delta_{t}}{a_{k}^{2}}\right) f(t)\right] d \mu(t)
$$

$$
=\int_{0}^{\infty} \frac{G_{N_{i}}^{*}(x, t)}{G_{N_{i}}^{*}(0, t)} G_{N_{i}}^{*}(0, t)\left[\prod_{k=1}^{N_{i}}\left(1-\frac{\Delta_{t}}{a_{k}^{2}}\right) f(t)\right] d_{\mu}(t) .
$$

Now let

$$
\alpha_{N_{i}}(x, t)=\frac{G_{N_{i}}^{*}(x, t)}{G_{N_{i}}^{*}(0, t)}
$$

and

$$
\beta_{N_{i}}(t)=\int_{0}^{t} G_{N_{i}}^{*}(0, t)\left[\prod_{k=1}^{N_{i}}\left(1-\frac{\Delta_{t}}{a_{k}^{2}}\right) f(t)\right] d \mu(t) .
$$

Then (1) becomes

$$
f(x)=\int_{0}^{\infty} \alpha_{N_{i}}(x, t) d \beta_{N_{i}}(t) .
$$

Note that since the integrand defining $\beta_{N_{i}}(t)$ is non-negative, $\beta_{N_{i}}(t) \uparrow$ and

$$
\int_{0}^{\infty} d \beta_{N_{i}}(t)=\beta_{N_{i}}(\infty)=f(0) \text {. }
$$

This implies that the $\beta_{N_{i}}(t)$ form a sequence of uniformly bounded functions which are of uniformly bounded total variation. Further, if we set

$$
\alpha(x, t)=\frac{G(x, t)}{G(0, t)},
$$

then, for each $x, \alpha(x, t) \uparrow$ by III, Theorem 4.4. Also, $\alpha(x, t)$ is a continuous function of $t$ for each $x$, and

$$
\alpha(x, \infty)=\mathscr{P}\left(a_{1} x\right) .
$$

The hypotheses of Theorem 4.2 are thus satisfied by $\alpha(x, t)$ and the $\beta_{N_{i}}(t)$, so that

$$
\lim _{N_{i_{j}} \rightarrow \infty} \int_{0}^{\infty} \alpha(x, t) d \beta_{N_{i_{j}}}(t)=\int_{0}^{\infty} \alpha(x, t) d \beta(t)+c \not{P}\left(a_{1} x\right),
$$

where

$$
\beta(t)=\lim _{N_{i_{j}} \rightarrow \infty} \beta_{N_{i_{j}}}(t)
$$


for every $t$ which is a point of continuity of $\beta(t)$. Clearly $\beta(t) \geqq 0$ and $\beta(t) \uparrow$. For $N$ sufficiently large, the $\alpha_{N_{i}}(x, t)$ are also continuous functions of $t, \alpha_{N_{i}}(x, t) \uparrow$ for fixed $x$ and $\alpha_{N_{i}}(x, \infty)=\mathscr{I}\left(a_{1} x\right)$. Further, by Lemma 4.1, we have that $\lim _{N \rightarrow \infty} \alpha_{N_{i}}(x, t)=\alpha(x, t)$ uniformly for $x$ fixed and for $t$ in every finite interval. Hence $\lim _{N \rightarrow \infty}\left\|\alpha_{N_{i}}(x, t)-\alpha(x, t)\right\|_{\infty}=0$ for each $x$. Now consider

$$
f(x)-\int_{0}^{\infty} \alpha(x, t) d \beta_{N_{i j}}(t)=\int_{0}^{\infty}\left[\alpha_{N_{i}}(x, t)-\alpha(x, t)\right] d \beta_{N_{i j}}(t),
$$

so that

$$
\begin{aligned}
\left|f(x)-\int_{0}^{\infty} \alpha(x, t) d \beta_{N_{i_{j}}}(t)\right| & \leqq \int_{0}^{\infty}\left|\alpha_{N_{i_{j}}}(x, t)-\alpha(x, t)\right| d \beta_{N_{i_{j}}}(t) \\
& \leqq\left\|\alpha_{N_{i_{j}}}(x, t)-\alpha(x, t)\right\|_{\infty} f(0) .
\end{aligned}
$$

Thus

$$
f(x)=\lim _{N_{i_{j}} \rightarrow \infty} \int_{0}^{\infty} \alpha(x, t) d \beta_{N_{i_{j}}}(t),
$$

or by (5), (7) and (8),

$$
\begin{aligned}
f(x) & =\int_{0}^{\infty} \alpha(x, t) d \beta(t)+c \mathscr{I}\left(a_{1} x\right) \\
& =\int_{0}^{\infty} G(x, t) d \psi(t)+c \mathscr{I}\left(a_{1} x\right),
\end{aligned}
$$

where

$$
\psi(t)=\int_{0}^{t} \frac{1}{G(0, u)} d \beta(u) .
$$

Theorem 2.1 establishes the fact that

$$
\int_{0}^{\infty} G(x, t) d \psi(t)=o\left(\mathscr{F}\left(a_{1} x\right)\right), \quad x \rightarrow \infty,
$$

and hypothesis (c), that $f(x)=o\left(\mathscr{P}\left(a_{1} x\right)\right), x \rightarrow \infty$, whence $c=0$, and we have $f(x)=\int_{0}^{\infty} G(x, t) d \psi(t)$, with $\psi(t) \uparrow$.

\section{BIBLIOGRAPHY}

1. C. Fox, The inversion of convolution transforms by differential operators, Proc. Amer. Math. Soc. 4 (1953), 880-887.

2. F. R. Gantmacher, The theory of matrices, Vols. 1, 2, Chelsea, New York, 1959.

3. R. R. Goldberg, Fourier transforms, Cambridge Tracts in Mathematics and Mathematical Physics, No. 52, Cambridge Univ. Press, New York, 1961. 
4. D. T. Haimo, Variation diminishing transformations, Bull. Amer. Math. Soc. 70 (1964), 271-274.

5. I. I. Hirschman, Jr., Variation diminishing Hankel transforms, J. Analyse Math. 8 (1960-61), 307-336.

6. Variation diminishing transformations and ultraspherical polynomials, J. Analyse Math. 8 (1960-61), 337-360.

7. , Variation diminishing transformations and orthogonal polynomials, J. Analyse Math. 9 (1961), 177-193.

8. , Variation diminishing transformations and Sturm-Liouville systems, Comment. Math. Helv. 36 (1961), 214-233.

9. Variation diminishing transformations and general orthogonal polynomials, Canad. J. Math. 16 (1964), 98-107.

10. I. I. Hirschman, Jr. and D. V. Widder, The convolution transform, Princeton Univ. Press, Princeton, N. J., 1955.

11. I. P. Natanson, Theory of functions of a real variable, Vols. I, II, Ungar, New York, 1955.

12. G. Pólya and G. Szegö, Aufgaben und Lehrsatze aus der Analysis, Dover, New York, 1945.

13. I. J. Schoenberg, On totally positive functions, Laplace integrals and entire functions of the Laguerre-Polya-Schur type, Proc. Nat. Acad. Sci. U.S.A. 33 (1947), 11-17.

14. , On variation diminishing integral operators of the convolution type, Proc. Nat. Acad. Sci. U.S.A. 34 (1948), 164-169.

15. E. C. Titchmarsh, The theory of functions, 2nd ed., Oxford Univ. Press, Oxford, 1939. 16. - Introduction to the theory of Fourier integrals, Oxford Univ. Press, Oxford, 1948.

17. G. N. Watson, $A$ treatise on the theory of Bessel functions, 2nd ed., Cambridge Univ. Press, Cambridge, 1958.

18. D. V. Widder, Advanced calculus, 2nd ed., Prentice-Hall, Englewood Cliffs, N. J., 1961. 19. _ The Laplace transform, Princeton Mathematical Series, Vol. 6, Princeton Univ. Press, Princeton, N. J., 1941.

\section{SOUTHERN ILLINOIS UNIVERSITY,}

ALton, Illinois 